\title{
Continuous administration of the p38 $\alpha$ inhibitor neflamapimod during the subacute phase after transient ischemia-induced stroke in the rat promotes dose-dependent functional recovery accompanied by increase in brain BDNF protein level
}

John Alam ${ }^{1 *}$, Michael Krakovsky ${ }^{2}$, Ursula Germann¹, Aharon Levy ${ }^{2}$

${ }^{1}$ EIP Pharma, Inc., Boston, Massachusetts, United States of America

${ }^{2}$ Pharmaseed Ltd., Ness-Ziona, Israel

* Corresponding author

Email: jalam@eippharma.com 


\section{PAGE 2}

\section{Abstract}

There is unmet need for effective stroke therapies. Numerous neuroprotection attempts for acute cerebral ischemia have failed and there is growing interest in developing therapies that widen the treatment initiation window and promote functional recovery through increasing synaptic plasticity. The p38 $\alpha$ mitogen-activated protein kinase is an already proven target for acute experimental stroke intervention and was hypothesized to also contribute to neuroinflammationmediated impairment of recovery during the subacute phase. Neflamapimod, an orally bioavailable, brain-penetrant, potent and selective small molecule p38 $\alpha$ inhibitor was evaluated as a subacute phase stroke treatment to promote recovery in this research study. Neflamapimod administration at two clinically relevant dose levels was initiated outside of the previously characterized neuroprotection window of less than 24 hours after stroke for $\mathrm{p} 38 \alpha$ inhibitors to rats after transient middle cerebral artery occlusion. Continuous administration of neflamapimod, starting at 48 hours after reperfusion, significantly improved behavioral outcomes assessed by the modified neurological severity score at four- and six-weeks post stroke in a dose-dependent manner. Neflamapimod also demonstrated beneficial effects on additional measures of sensory and motor function and resulted in a dose-related increase in the terminal brain-derived neurotrophic factor protein level in both the injured and uninjured brain hemisphere. Variable interleukin-1 $\beta$ levels were detected in the injured brain hemisphere at study termination in a subset of the animals within every test group, implying ongoing, chronic inflammation, however, no clear neflamapimod effect on interleukin-1 $\beta$ production was observable. The dose-related in vivo efficacy of neflamapimod offers the possibility of both expanding the window for initiation of therapy after stroke and for improving recovery after a completed stroke. Since neflamapimod is already in mid-stage clinical trials for Alzheimer's disease 
PAGE 3

and related dementias, the current results make it especially attractive for evaluation in a proof-ofconcept clinical trial as therapeutic to promote recovery after ischemic stroke.

\section{Introduction}

Stroke is a frequent cause of death as well as a leading cause of acquired disability worldwide and is associated with a substantial economic burden due to high costs for treatment and post-stroke care $[1,2]$. Approximately $80 \%$ of strokes are ischemic in nature due to thromboembolic occlusion of a major artery or its branches, leading to a cascade of events that causes irreversible tissue injury [3]. The only approved medical treatments for acute ischemic stroke are intravenous thrombolysis with recombinant tissue plasminogen activator (TPA), resulting in recanalization of occluded vessels if applied within a short time period (up to 4.5 hours) after stroke [4], or

thrombectomy, ideally performed within 6 hours after stroke symptom onset (although under certain conditions beneficial if applied within 24 hours after stroke symptom onset), involving mechanical removal of the thromboembolic material occluding the vessel lumen [5].

Based on pathological characteristics and their timing, a stroke is classified into three clinical phases, including the acute (i.e. first 48 hours after stroke onset), the subacute (from 48 hours to $>6$ weeks post stroke) and the chronic phase (starts at 3-6 months post stroke) [6]. The acute phase represents an opportunity to salvage threatened tissue and reduce the extent of injury, for example via reperfusion or neuroprotection $[7,8]$. The subacute phase represents the recovery stage, which is characterized by brain repair initiation, so therapeutic strategies include enhancing the underlying spontaneous recovery processes, modifying inflammation, lifting diaschisis, or reducing late neuronal death $[7,8]$. 


\section{PAGE 4}

Numerous attempts at providing neuroprotection during the acute phase of stroke have failed and currently, there is no United States Food and Drug Administration (FDA)-approved agent for neuroprotection after ischemic stroke [9-11]. Clearly there is an urgent need for alternative, more widely applicable treatment options for ischemic stroke including therapeutics that enable expansion of the time window from stroke to treatment. While it remains important to continue to advance novel acute stroke interventions, there is also high interest in the development of novel therapies that are directed at promoting functional recovery from stroke via increasing neuronal and synaptic plasticity during the subacute phase $[8,9,12]$. The goal is to identify disease-modifying treatments that can be administered after the acute phase of stroke is complete $[8,9,12]$. It is envisioned that such neurorestorative agents are administered during the subacute and/or the chronic phase post stroke only and that they may target compromised cerebral tissue and/or surrounding intact tissue to promote brain plasticity $[8,12]$.

The p38 mitogen-activated protein kinase (MAPK) pathway plays a crucial role in several central nervous system (CNS) disorders, including Alzheimer's disease, Down's syndrome, Parkinson's disease, spinal cord injury (SCI), and cerebral ischemia [13, 14]. Among the four p38 MAPK family members, p38 $\alpha$ was discovered first as a stress-activated protein kinase that plays a central role in inflammation $[15,16]$. It is also the best characterized isoform as a target for CNS drug discovery $[17,18]$. A large variety of biological roles have been attributed to $\mathrm{p} 38 \alpha$ in brain pathology which depend on the type and stage of the CNS disorder, the brain region, the cell type, and include modulation of pro-inflammatory cytokine (e.g. IL-1 $\beta$ and tumor necrosis factor $\alpha$ $(\mathrm{TNF} \alpha)$ ) production as well as signaling (e.g. in glia, microglia, astrocytes, neurons) in the brain, and orchestration of neurotoxicity, neuroinflammation and/or synaptic dysfunction [14, 17-19]. 


\section{PAGE 5}

In the adult mouse, $\mathrm{p} 38 \alpha$ is highly expressed in different brain areas including cerebral cortex, hippocampus, cerebellum, and a few nuclei of the brainstem [20]. At the subcellular level, p38 $\alpha$ is distributed in dendrites and in cytoplasmic and nuclear regions of the cell body of neurons [20]. Activation of p38 MAPK, including p38 $\alpha$, signaling after experimental ischemic stroke in rodents has been demonstrated in neurons, astrocytes and microglia [21-25], and p38 $\alpha$ has been established as a driver of neuroinflammation-mediated cell death in the acute phase of ischemic stroke [26, 27]. Therefore, several inhibitors of p38 MAPK that exhibit different potency and kinase selectivity, all of them most potently blocking $\mathrm{p} 38 \alpha$ versus other p38 isoforms (p38 $\beta, \mathrm{p} 38 \gamma, \mathrm{p} 38 \delta$ ) have been administered during the acute phase with treatment initiation within the first 24 hours after stroke in experimental models of cerebral ischemia, and all of them have provided robust neuroprotection [25, 28-32].

While p $38 \alpha$ has already been proven as a therapeutic target for acute stroke intervention, it is unclear whether $\mathrm{p} 38 \alpha$ also plays a critical role in impairing functional recovery during the subacute phase of stroke which embodies brain repair initiation [6-8]. Time course analyses of phospho-p38 (i.e. the activated from of $\mathrm{p} 38$ ) expression in experimental rat stroke models reported increased phospo-p38, including phospho-p38 $\alpha$, immediately after cerebral ischemia within neurons and other cell types, and, despite some fluctuations observed in some of the studies, also demonstrated that phospho-p38 $\alpha$ remained elevated and associated with neurons, as well as other cell types, for $>4$ days, and in one study up to 14 days, implying that p38 $\alpha$-activation during the subacute phase could be partly responsible for poor recovery from stroke [22, 24, 25, 31]. A one week study of a novel p38 $\alpha$ inhibitor, the tetra-substituted thiophene VCP979, evaluated as a treatment of phothothrombotic ischemic stroke in streptozocin-induced Type 2 diabetes mellitus (T2DM) mice demonstrated beneficial efficacy resulting in neuroprotection as well as axonal/white matter 


\section{PAGE 6}

remodeling within the motor cortex [32]. Whereas this study offered novel mechanistic insights for a p38 $\alpha$ inhibitor that are relevant for brain repair, it did not address widening the window to treatment initiation since VCP979 administration started at 24 hours post stroke [32], at a time point that is within the acute phase of photothrombotic stroke in mice $[33,34]$.

Brain-derived neurotrophic factor (BDNF) promotes neural plasticity and recovery after stroke [35] and the proinflammatory cytokine interleukin-1 beta (IL-1 $\beta$ ) is upregulated after ischemic stroke [36-40]. In subacute/chronic inflammatory conditions, IL-1 $\beta$ is known to be a key component of the inflammatory response in the brain that mediates neurodegenerative effects of inflammation on cognition and synaptic plasticity [41]. Chronic elevation of IL-1 $\beta$, such as IL-1 $\beta$ elevation in the aging brain, suppresses BDNF production [42], and in vitro it has been shown that increased IL-1 $\beta$ inhibits BDNF effects on neuronal/synaptic plasticity via induction of p38 MAPK [43]. All the above-described research findings taken together stimulated the idea to investigate whether neflamapimod, a blood brain barrier-penetrant small molecule p38 $\alpha$ inhibitor, may have beneficial efficacy as a subacute phase stroke treatment via promoting functional recovery through increasing synaptic plasticity.

The pyrimido pyridazine neflamapimod (International Non-proprietary Name for molecule previously code-named VX-745) is a potent, highly selective, ATP-competitive inhibitor of p38 $\alpha$ that yields higher CNS than peripheral blood exposure after oral administration [44, 45]. It exhibits efficacy and safety profiles in preclinical species to merit ongoing clinical investigation for CNS disorder indications [45]. Neflamapimod is currently being evaluated in the clinic for its potential to reverse synaptic dysfunction in three different CNS disorders (Alzheimer's disease, Huntington's disease, dementia with Lewy bodies). The first two exploratory clinical studies of neflamapimod in 9 and 16 subjects, respectively, with mild cognitive impairment due to Alzheimer's disease 


\section{PAGE 7}

demonstrated that neflamapimod penetrates the blood-brain barrier in humans and exhibits diseaserelevant pharmacological activity in the brain $[45,46]$.

In a previous research study [47], neflamapimod was evaluated in 20- to 24-month old Fischer rats with cognitive deficits resulting from IL-1 $\beta$-induced impairment of synaptic plasticity [48]. Results showed that a very low dose of neflamapimod (1.5 mg/kg administered twice daily) reversed spatial learning deficits in the Morris-Water-Maze test with no effects on hippocampal IL$1 \beta$ levels, and that a 3 -fold higher dose $(4.5 \mathrm{mg} / \mathrm{kg}$ administered twice daily) reduced IL-1 $\beta$ levels in the hippocampus but did not result in pro-cognitive effects [47]. These results suggested that the neflamapimod pro-cognitive effects were mediated through decreasing IL-1 $\beta$ signaling in hippocampal neuron target cells, rather than through reducing IL-1 $\beta$ production [47]. Thus, the present study was also undertaken to extend the aged rat behavioral results by investigating these two clinically relevant neflamapimod dose levels in a subacute phase treatment experimental paradigm for their efficacy in promoting neurologic plasticity and functional recovery in experimental stroke.

In recognition of the unmet need for stroke therapies that enable a wider treatment window and show potential for promoting functional recovery via beneficial effects on synaptic plasticity, the objectives of the present study were (1) to evaluate neflamapimod at clinically relevant dose levels for its in vivo efficacy to promote neurologic recovery as assessed by the modified neurological severity score (mNSS) and additional behavioral tests in a rat transient middle cerebral artery occlusion (tMCAO) model; (2) to initiate administration of neflamapimod outside the known neuroprotection window for $\mathrm{p} 38 \alpha$ inhibitors and address the possibility for widening the window for treatment post stroke induction; (3) to assess the neurogenic factor BDNF in the brain as a potential biomarker for monitoring neflamapimod effects on synaptic plasticity; and (4) to measure IL-1 $\beta$ 
PAGE 8

levels as an inflammatory biomarker in the brain after the treatment period to gauge whether chronic inflammation may play a role in this experimental stroke model and whether a neflamapimod antiinflammatory effect may be detectable.

\section{Materials and methods}

\section{Animals and general health monitoring}

Seventy-six young (3-month old) male Sprague Dawley rats (Harlan Laboratories, Israel) weighing $328 \mathrm{~g} \pm 20 \%$ were included in the study. The protocol for the study was approved by the Israeli Animal Care and Use Committee (approval number IL-15-01-15) and was conducted in accordance with the Israeli guidelines that conform to the United States Public Health Service's Policy on Humane Care and Use of Laboratory Animals. Animal handling was performed according to guidelines of the National Institute of Health $(\mathrm{NIH})$ and the Association for Assessment and Accreditation of Laboratory Animal Care (AAALAC). To assess the health status of the animals throughout the study, the general health status was monitored daily and body weight was determined weekly.

\section{Transient middle cerebral artery occlusion}

Transient middle cerebral artery occlusion (tMCAO) in the right brain hemisphere was performed on Day 1 according to the method of Longa et al. [49], in which a $4 \mathrm{~cm}$ length of 4-0 monofilament nylon suture is inserted under anesthesia through the proximal external carotid artery into the internal carotid artery and thence into the circle of Willis, effectively occluding the middle cerebral artery. Anesthesia was induced with $4 \%$ isoflurane in a mixture of $70 \% \mathrm{~N} 2 \mathrm{O}$ and $30 \% \mathrm{O} 2$ 
PAGE 9

and maintained with $1.5-2 \%$ isoflurane. The surgical wound was subsequently closed, and the animals were returned to their cages to recover from anesthesia. Two hours after occlusion rats were re-anesthetized, monofilament was withdrawn to allow reperfusion, surgical wound was closed, and rats were returned to their cages.

\section{Neurologic scoring and behavioral evaluations}

The individual performing neurologic scoring and behavioral assessments was unaware of the group assignments, thus, performed blinded evaluations. Neurologic scoring by mNSS evaluation according to Chen et al. [50] was performed at least one day before the Day 1 tMCAO, and on Day 2, at Week 4, and at Week 6 following tMCAO. The mNSS values included the results of a set of clinical-neurological tests, a composite of motor, sensory, reflex and balance tests and well-defined score values (summarized in Table 1 [50]), that were used to assess the effect of neflamapimod compared to vehicle control. Taking into account the different tests and score values described in Table 1, the mNSS was graded on a composite scale of 0 to 18 , in which the normal, healthy animal value was 0 and the maximal deficit value after tMCAO was represented by 18 [50]. An overall mNSS value of $\geq 10$ was predefined as an inclusion criterion for enrolling a rat with stroke into the neflamapimod treatment study.

Table 1. Modified Neurological Severity Score (mNSS) tests and scoring values [50].

\begin{tabular}{|l|l|}
\hline \multicolumn{2}{|l|}{ Motor test score values and descriptions } \\
$($ Normal score $=\mathbf{0}$; maximum possible summary score $=\mathbf{6})$ \\
\hline 0 or $1^{*}$ & Flexion of forelimb after raising rat by the tail \\
\hline 0 or $1^{*}$ & Flexion of hindlimb after raising rat by the tail \\
\hline
\end{tabular}




\begin{tabular}{|c|c|}
\hline 0 or $1 *$ & Head moved $>10^{\circ}$ to vertical axis within 30 seconds after raising rat by the tail \\
\hline 0 & Normal walk after placing rat on the floor \\
\hline 1 & Inability to walk straight after placing rat on the floor \\
\hline 2 & Circling toward paretic side after placing rat on the floor \\
\hline 3 & Falls down to paretic side after placing rat on the floor \\
\hline \multicolumn{2}{|c|}{$\begin{array}{l}\text { Sensory test score values and descriptions } \\
(\text { Normal score }=0 ; \text { maximum possible summary score }=2)\end{array}$} \\
\hline 0 or $1 *$ & Placing test (visual and tactile test) \\
\hline 0 or $1 *$ & $\begin{array}{l}\text { Procioceptive test (deep sensation, pushing paw against table to stimulate limb } \\
\text { muscles) }\end{array}$ \\
\hline \multicolumn{2}{|c|}{$\begin{array}{l}\text { Beam and balance test score values and descriptions } \\
(\text { Normal score }=0 \text {; maximum possible summary } \text { score }=6)\end{array}$} \\
\hline 0 & Balances with steady posture \\
\hline 1 & Grasps side of beam \\
\hline 2 & Hugs beam and 1 limb falls down from beam \\
\hline 3 & Hugs beam and 2 limbs fall down from beam, or spins on beam (60 seconds) \\
\hline 4 & Attempts to balance on beam, but falls off ( $>40$ seconds) \\
\hline 5 & Attempts to balance on beam, but falls off ( $>20$ seconds) \\
\hline 6 & Falls off; no attempt to balance or hang on to beam $(<20$ seconds $)$ \\
\hline \multicolumn{2}{|c|}{$\begin{array}{l}\text { Reflex absence and abnormal movements test score values and descriptions } \\
(\text { Normal score }=0 \text {; maximum possible summary score }=4)\end{array}$} \\
\hline 0 or $1^{*}$ & Pinna reflex (head shakes when auditory meatus is touched with cotton) \\
\hline 0 or $1 *$ & Corneal reflex (eye blink when cornea is lightly touched with cotton) \\
\hline
\end{tabular}


PAGE 11

\begin{tabular}{|l|l|}
\hline 0 or $1^{*}$ & Startle reflex (motor response to a brief noise from snapping a clipboard paper) \\
\hline 0 or $1^{*}$ & Seizure, myoclonus, myodystony \\
\hline
\end{tabular}

*Score value of 1 was given for the inability to perform a test, or for the lack of a tested reflex, or for abnormal movement [50].

Additional behavioral evaluations included stepping test, body swing test, and forelimb placement test that were performed before the tMCAO procedure, and at Week 4 and Week 6 following tMCAO.

A stepping test was utilized to assess forelimb akinesia according to Pharmaseed's internal protocol. Each rat was held with its hind limbs fixed in one hand and the forelimb, not to be monitored, in the other, while the unrestrained forepaw touched the table. The number of adjusting steps of the forelimb to be monitored was counted while the animal was moved sideways along the table surface in the forehand and backhand direction over a distance of $85 \mathrm{~cm}$ during approximately five seconds. The stepping test was completed for both the left and right forelimbs at all time points indicated above.

For the body swing test, each rat was held approximately one inch from the base of its tail. It was then elevated to an inch above a surface of the table. The rat was held in the vertical axis, defined as no more than $10^{\circ}$ to either the left or the right side. A swing was recorded whenever the rat moved its head out of the vertical axis to either side. Before measuring another swing, the rat must have returned to the vertical position. Twenty (20) total swings were counted. A normal rat typically had an equal number of swings to either side. Adjusted body swing scores were calculated as the difference between leftward and rightward swings (i.e. the number of rightward swings subtracted from leftward swings). 
PAGE 12

For the forelimb-placing test, the examiner held the rat close to a tabletop and scored the rat's ability to place the forelimb on the tabletop in response to whisker, visual, tactile, or proprioceptive stimulation. Separate sub-scores were obtained for each mode of sensory input and added to give total scores $(0=$ normal, $12=$ maximally impaired $)$. Scores were given in half-point increments: whisker placing (0-2); visual placing (forward (0-2) and sideways (0-2)); tactile placing (dorsal (0-2) and lateral (0-2)); proprioceptive placing (0-2).

\section{Administration of test articles}

Test articles, neflamapimod at two different dose levels in 1\% (w/v) Pluronic F108 vehicle, or vehicle (1\% (w/v) Pluronic F108) were supplied by EIP Pharma, Inc. and administered at a dosing volume of $5 \mathrm{ml} / \mathrm{kg}$ by oral gavage twice daily (7 AM and $7 \mathrm{PM}$ ) on 5.5 days (i.e. Sunday through Friday at 7 AM and Sunday through Thursday at 7 PM) every week for six weeks (i.e. from Day 3 until Day 42), starting at 48 hours post reperfusion.

\section{Sample collection and analysis}

On Day 44, two days following the final dosing of test article or vehicle, rats were sacrificed by $\mathrm{CO}_{2}$ inhalation. Brains were harvested from all animals and samples were divided into left and right hemisphere. Samples were weighed, immediately frozen in liquid nitrogen, and stored at $-80^{\circ} \mathrm{C}$. For the IL-1 $\beta$ and BDNF analyses, tissue samples were defrosted and homogenized in $1 \mathrm{ml} / 200 \mathrm{mg}$ tissue of $20 \mathrm{mM}$ Tris-HCL pH 7.4 containing a protease inhibitor cocktail $(50 \mu \mathrm{l} / \mathrm{ml})$. Samples were centrifuged at $10,000 \mathrm{x}$ g for 15 minutes at $4^{\circ} \mathrm{C}$. Clear supernatants were aliquoted $(150 \mu \mathrm{l} /$ aliquot $)$ and stored at $-80^{\circ} \mathrm{C}$ until enzyme linked immunosorbent assay (ELISA) for IL-1 $\beta$ or BDNF was performed. For IL-1 $\beta$ analysis, ELISA was performed using the Rat IL-1 beta/IL-1F2 Quantikine 
ELISA Kit (R\&D Systems, Minneapolis, MN) according to manufacturer's instructions. For BDNF analysis, ELISA was performed using the Solid Phase Sandwich Quantikine ELISA Kit (96-well strip plates, R\&D Systems, Canada) according to manufacturer's instructions. For IL-1 $\beta$ and BDNF, standards and samples were tested in duplicates. For IL-1 $\beta$ assay, all the results below the lower limit of quantification (LLOQ) were assigned the LLOQ value of $5 \mathrm{pg} / \mathrm{ml}$. For BDNF assay, all the results were above the LLOQ.

\title{
Statistical analysis
}

For body weight monitoring, mNSS or other behavioral evaluations, and IL-1 $\beta$ levels, statistical analysis was performed by two-way analysis of variance (ANOVA) for repeated measures, followed by Bonferroni post-hoc test to adjust for the multiple comparisons. For BDNF levels, Jonckheere-Terpstra test was utilized to assess for treatment-dependent dose effect across placebo, $1.5 \mathrm{mg} / \mathrm{kg}$, and $4.5 \mathrm{mg} / \mathrm{kg}$ dose groups. In addition, one-way ANOVA followed by Bonferroni's multiple comparison test was performed to compare individual neflamapimod dose groups to the placebo group. One-way ANOVA was also used to compare the mNSS values at two different time points (e.g. Week 4 versus Day 2, or Week 6 versus Week 4) within an individual test group.

\section{Results}

\section{Robust experimental stroke induction in rats caused significant}

\author{
increase in mNSS value and low mortality during the acute phase (i.e.
}

\section{0-48 hours) after stroke}


A frequently used method, tMCAO, was used to generate a robust experimental stroke as assessed by mNSS functional testing or mortality. Ischemia was induced on Day 1 in 76 young adult, 3-month old male Sprague-Dawley rats by occlusion of the right middle cerebral artery, which was released after 2 hours [49]. Nine animals were lost during the first day and four additional animals were lost during the second day after the procedure, resulting in $\sim 17 \%$ mortality during the first 48 hours post tMCAO attributable to the severity of these rat's stroke.

On Day 2, at 24 hours after reperfusion, 67 surviving animals were subjected to neurological evaluation using the mNSS value (see Table $1[50]$ ) that rates neurological functioning on a scale from 0 (healthy) to 18 (maximum impairment) [51]. The measured mNSS value in individual rats rose from 0 before the Day $1 \mathrm{tMCAO}$ procedure to a mean \pm standard deviation (SD) value of 14.0 \pm 1.4 on Day 2, with individual animal mNSS values ranging from 12 to 17 , indicating successful induction of a robust experimental stroke.

On Day 3, at 48 hours post stroke, a total of 63 animals remained available for inclusion in the study and the animals ( $\mathrm{n} \geq 20$ per group at randomization) were randomized based on Day 2 mNSS values and assigned to one of three treatment groups: twice daily $1.5 \mathrm{mg} / \mathrm{kg}$ neflamapimod (i.e. 22 rats), twice daily $4.5 \mathrm{mg} / \mathrm{kg}$ neflamapimod (i.e. 21 rats), or twice daily vehicle (i.e. 20 control rats) during 5.5 days every week for a total of six weeks.

\title{
Observations of animal health preservation including body weight gain
}

\section{after initiation of neflamapimod or vehicle treatment during the}

\author{
subacute phase after stroke
}


PAGE 15

The first administration of neflamapimod treatment or vehicle control administration occurred on Day 3 at 48 hours post reperfusion. Overall animal health throughout the study was assessed by weekly body weight determinations, daily health assessment and survival observations. Three additional rats, including one animal from the $1.5 \mathrm{mg} / \mathrm{kg}$ neflamapimod dose group and two vehicle control rats, did not survive the first two weeks after initiation of dosing. Since these additional deaths were inversely related to the dose of neflamapimod, they were attributed to the severity of their stroke. These late mortalities indicate that the recovery process after a late treatment initiation in this rat tMCAO model was very slow during the first two weeks post-stroke. The 60 remaining rats $(\mathrm{n}=21$ in the $1.5 \mathrm{mg} / \mathrm{kg}$ and $\mathrm{n}=21$ in the $4.5 \mathrm{mg} / \mathrm{kg}$ neflamapimod groups, respectively, and $n=18$ in the vehicle group) completed the planned six weeks of dosing and were included in the neurologic and behavioral evaluations at Week 4 and Week 6.

Although the three experimental treatment groups were randomized based on Day 2 mNSS values, a retrospective analysis of the Day 1 mean \pm SD body weight data for the animals assigned to each group revealed that their body weights were also well balanced $(328.5 \pm 11.3 \mathrm{~g}$ for the $1.5 \mathrm{mg} / \mathrm{kg}$ and $325.3 \pm 11.1 \mathrm{~g}$ for the $4.5 \mathrm{mg} / \mathrm{kg}$ neflamapimod treatment group, respectively, and $328.2 \pm 9.6 \mathrm{~g}$ for the vehicle control group) as shown in Table 2 . Weekly body weight monitoring and two-way ANOVA statistics followed by Bonferroni post-hoc comparisons revealed no statistically significant differences in body weight for the three study groups at any time throughout the study. Additionally, no differences in general clinical signs were observed upon daily animal health monitoring (data not shown). The mean \pm SD body weight for the 3-month old rats was $327.0 \pm 10.9 \mathrm{~g}$ on Day 1 and all surviving animals had a mean \pm SD body weight $418.3 \pm 27.6 \mathrm{~g}$ at Week 6 , and a similar body weight gain of $\sim 27 \%$ was observed in all three test groups throughout the study period (Table 2 ). These observations together with favorable results for all the daily health assessments point out that the 
neflamapimod and vehicle treatments were generally well-tolerated, providing no treatment-related adverse clinical signs. Moreover, these findings exclude the possibility that a difference in the general health of the rats contributed to neflamapimod treatment-mediated effects on functional recovery when compared to the effects observed in the vehicle-treated group.

Table 2. Body weight monitoring of the three treatment groups, including vehicle control, 1.5 $\mathrm{mg} / \mathrm{kg}$ neflamapimod (NFMD), $4.5 \mathrm{mg} / \mathrm{kg}$ NFMD throughout the study.

\begin{tabular}{|l|c|c|c|}
\hline Body weight & Body weight & Body weight & Body weight \\
& Vehicle & $\mathbf{1 . 5} \mathbf{~ m g} / \mathbf{k g}$ NFMD & $\mathbf{4 . 5} \mathbf{~ m g / k g ~ N F M D ~}$ \\
& Mean \pm SD (g) & Mean \pm SD (g) & Mean \pm SD (g) \\
\hline Day 1 & $328.2 \pm 9.6$ & $328.5 \pm 11.3$ & $325.3 \pm 11.1$ \\
\hline Week 1 & $309.2 \pm 36.3$ & $325.7 \pm 26.8$ & $321.1 \pm 27.2$ \\
\hline Week 2 & $342.2 \pm 34.2$ & $353.9 \pm 24.9$ & $353.3 \pm 23.8$ \\
\hline Week 3 & $369.2 \pm 30.7$ & $378.5 \pm 23.1$ & $374.8 \pm 21.4$ \\
\hline Week 4 & $388.6 \pm 30.5$ & $397.3 \pm 25.1$ & $393.9 \pm 25.5$ \\
\hline Week 5 & $403.6 \pm 31.4$ & $412.4 \pm 25.6$ & $407.5 \pm 21.1$ \\
\hline Week 6 & $415.0 \pm 34.5$ & $421.5 \pm 26.8$ & $417.8 \pm 23.0$ \\
\hline
\end{tabular}

Dose-related improvement in neurologic and behavioral mNSS after initiation of neflamapimod versus vehicle treatment during the

\author{
subacute phase post stroke
}


Neurologic and behavioral improvements are reliable parameters for measuring the therapeutic efficacy in experimental stroke and the mNSS is one of the most commonly used neurological scales in experimental stroke studies that includes a composite of motor (muscle status and abnormal movement), sensory (visual, tactile, and proprioceptive), balance and reflex tests (see Table 1 ) on a scale up to 18 for rats $[50,51]$. On Day 2 , the mean \pm SD values for $\mathrm{mNSS}$ were similar across the groups of stroked rats, indicating that the three randomized experimental groups were balanced a day prior to treatment initiation (Fig 1A). The measured mNSS mean \pm SD values on Day 2 were $13.9 \pm 1.1$ in the $1.5 \mathrm{mg} / \mathrm{kg}$ and $13.9 \pm 1.4$ in the $4.5 \mathrm{mg} / \mathrm{kg}$ neflamapimod dose group, and $14.3 \pm 1.7$ in the vehicle control group. In light of the maximum possible mNSS value of 18 (Table 1), all animals exhibited severe neurologic deficits prior to neflamapimod treatment initiation.

\section{Fig 1. Dose-related neflamapimod (NFMD) subacute treatment effects on mNSS of rats with}

tMCAO. The study included three groups: vehicle (Pluronic F108; n=18), 1.5 mg/kg neflamapimod $(\mathrm{n}=21)$, and $4.5 \mathrm{mg} / \mathrm{kg}$ neflamapimod (n=21). (A) Mean \pm SD mNSS value at Day 2, Week 4 and Week 6. Both at Week 4 and Week 6, the mean \pm SD mNSS values were decreased and statistically significant lower in each neflamapimod dose group when compared to vehicle. In addition, a dose response effect on improvement in neurologic function was indicated by the lower mean \pm SD mNSS value in the $4.5 \mathrm{mg} / \mathrm{kg}$ dose group when compared to that of the $1.5 \mathrm{mg} / \mathrm{kg}$ dose group. (B) Absolute changes in mNSS \pm SD from Day 2 to Week 4 and Day 2 to Week 6. Statistically significant mNSS differences were observed at both time point comparisons for vehicle compared to 1.5 or $4.5 \mathrm{mg} / \mathrm{kg}$ dose groups. Significance of between group differences was assessed using a two-way analysis of variance (ANOVA) with a Bonferroni correction $(* P<0.05 ; * * P<0.01 ; * * * P<0.001)$. 


\section{PAGE 18}

Importantly, clear differences were demonstrated between the groups treated with neflamapimod compared to the vehicle-treated group. As shown in Fig 1A, the mean \pm SD mNSS values were statistically significantly lower $(P<0.001$ for all comparisons $)$ in the $1.5 \mathrm{mg} / \mathrm{kg}$ dose group (10.0 \pm 0.7 at Week 4 and $9.4 \pm 0.7$ at Week 6$)$ and in the $4.5 \mathrm{mg} / \mathrm{kg}$ dose group $(9.1 \pm 0.7$ at Week 4 and $8.5 \pm 1.0$ at Week 6 ) at both time points when compared to vehicle (see above, $12.1 \pm 1.1$ at Week 4 and $11.4 \pm 0.9$ at Week 6). These results document a positive effect of neflamapimod on neurologic repair following induction of ischemic stroke. Furthermore, a dose-related effect on improvement in neurologic function was indicated by the statistically significant lower mean mNSS in the $4.5 \mathrm{mg} / \mathrm{kg}$ dose group when compared to the $1.5 \mathrm{mg} / \mathrm{kg}$ dose group $(P<0.05$ at Week 4 and Week 6). Findings were similar when absolute changes in mNSS from Day 2 to Week 4 and Day 2 to Week 6 in the $1.5 \mathrm{mg} / \mathrm{kg}$ dose group (3.9 and 4.5, respectively) and the $4.5 \mathrm{mg} / \mathrm{kg}$ dose group (4.8 and 5.4, respectively) were compared to the absolute changes in the vehicle group (2.1 and 2.7, respectively), as summarized in Fig 1B. Statistically significant differences in absolute change in mNSS were observed at both time point comparisons for the $1.5 \mathrm{mg} / \mathrm{kg}$ dose group $(P<0.01)$ and the $4.5 \mathrm{mg} / \mathrm{kg}$ dose group $(P<0.001)$ compared to the vehicle control group.

It is also noteworthy that within all three individual groups the mean mNSS values were statistically significantly $(P<0.001)$ decreased at the Week 4 and Week 6 time points versus Day 2 . The vehicle group results for the mean \pm SD mNSS values (12.1 \pm 1.1 at Week 4 and $11.4 \pm 0.9$ at Week 6 versus $14.3 \pm 1.7$ on Day 2 ) imply a low degree of spontaneous recovery of neurologic functions in these study animals from Day 2 to Week $4(P<0.001)$, as well as from Week 4 to Week $6(P<0.05)$. It should be noted, however, that the mean mNSS value of the vehicle-treated control group decreased by $\sim 19 \%$ only during the six-week study period, and the mean mNSS score of 11.4 at 
Week 6 was nowhere close to the pre-stroke baseline mNSS value of 0 for healthy animals. Hence, the underlying spontaneous recovery process was very slow in this model.

\section{Neflamapimod-mediated improvement in motor and sensory function}

\section{behavioral tests during the subacute stroke phase}

Additional behavioral evaluations of motor and sensory functions (stepping test, body swing and forelimb placement) were performed to complement the mNSS end point for assessment of neflamapimod treatment effects during the subacute phase post stroke. The findings from these additional tests are presented in Fig 2 and were consistent with results of the mNSS evaluation.

Fig 2. Effects of four- and six-week neflamapimod (NFMD) subacute treatment on stepping, body swing and forelimb placement tests of rats with tMCAO. Treatment groups include vehicle $(\mathrm{n}=18), 1.5 \mathrm{mg} / \mathrm{kg}$ neflamapimod $(\mathrm{n}=21)$, and $4.5 \mathrm{mg} / \mathrm{kg}$ neflamapimod $(\mathrm{n}=21)$. (A) Stepping test. Rats in both neflamapimod dose groups took a statistically significant greater mean \pm SD number of left forelimb steps at Week 4 and Week 6 compared to vehicle-treated animals. (B) Body swing test. Mean \pm SD adjusted body swing score values at Week 4 and Week 6 in the $1.5 \mathrm{mg} / \mathrm{kg}, 4.5 \mathrm{mg} / \mathrm{kg}$ dose groups were statistically significantly lower when compared to vehicle group. (C) Forelimb placement test. Mean \pm SD forelimb placement scores at Week 4 and Week 6 in the $1.5 \mathrm{mg} / \mathrm{kg}$ and $4.5 \mathrm{mg} / \mathrm{kg}$ dose groups were statistically significantly lower when compared to vehicle group.

Statistical significance of between group differences was assessed using ANOVA with a Bonferroni correction $(* P<0.05 ; * * P<0.01 ; * * * P<0.001)$. 


\section{PAGE 20}

In the left forelimb stepping test, rats in both neflamapimod dose groups had a statistically significant $(P<0.001$ in all comparisons at a given time point) greater mean \pm SD number of steps at

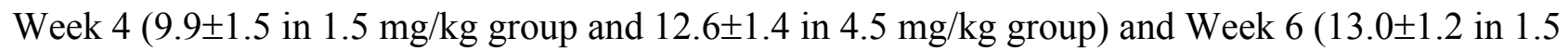
$\mathrm{mg} / \mathrm{kg}$ group and $14.4 \pm 1.4 \mathrm{in} 4.5 \mathrm{mg} / \mathrm{kg}$ group) compared to vehicle-treated animals $(7.6 \pm 1.3$ at Week 4 and 8.7 \pm 1.6 at Week 6) as shown in Fig 2A. Supporting that the effects of neflamapimod were specific to neurologic recovery following tMCAO, the mean \pm SD number of steps for the right forelimbs were similar in all groups at Week $4(19.1 \pm 0.4$ for $1.5 \mathrm{mg} / \mathrm{kg}, 19.1 \pm 0.4$ for $4.5 \mathrm{mg} / \mathrm{kg}$ and 18.9.7 \pm 0.2 for vehicle group) and Week $6(19.1 \pm 0.4$ for $1.5 \mathrm{mg} / \mathrm{kg}, 19.2 \pm 0.4$ for $4.5 \mathrm{mg} / \mathrm{kg}$ and 19.1 \pm 0.2 for vehicle group), respectively, and comparable to pre-stroke baseline left forelimb (19.8 \pm 0.6 for $1.5 \mathrm{mg} / \mathrm{kg}, 18.8 \pm 3.3$ for $4.5 \mathrm{mg} / \mathrm{kg}$ and $19.7 \pm 0.8$ for vehicle group) or baseline right forelimb (20.0 \pm 0.7 for $1.5 \mathrm{mg} / \mathrm{kg}, 20.1 \pm 0.7$ for $4.5 \mathrm{mg} / \mathrm{kg}$ and $19.7 \pm 0.8$ for vehicle group) values.

Additional results from the body swing (BSW) tests indicate a more prominent motor recovery in neflamapimod-treated animals. The mean \pm SD adjusted BSW score in the tests at Week 4 and Week 6 in the $1.5 \mathrm{mg} / \mathrm{kg}(6.8 \pm 4.6$ and $5.6 \pm 4.2$, respectively) and $4.5 \mathrm{mg} / \mathrm{kg}(2.8 \pm 3.9$ and $1.1 \pm 2.9$, respectively) dose groups were all statistically significantly $(P<0.001)$ lower when compared to vehicle group (15.9 \pm 3.0 and $16.2 \pm 3.6$, respectively) at the same time points, as presented in Fig 2B.

Finally, the mean \pm SD forelimb placement scores in the forelimb placement tests at Week 4 and Week 6 in the $1.5 \mathrm{mg} / \mathrm{kg}(8.3 \pm 1.2$ and $5.5 \pm 2.2$, respectively) and $4.5 \mathrm{mg} / \mathrm{kg}(6.0 \pm 2.1$ and $3.1 \pm 1.7$, respectively) neflamapimod dose groups were also statistically significantly $(P<0.01$ in all comparisons at the two time points) lower when compared to vehicle group $(10.9 \pm 1.1$ and $10.1 \pm 0.9$, respectively), as shown in Fig $2 \mathrm{C}$. The forelimb placement test scored the above-mentioned 
mean \pm SD forelimb placement scores positive values for the affected left forelimbs post stroke only. All the forelimb placement test scores for the left and right forelimbs at baseline, as well as all the values for the right forelimbs at Week 4 and Week 6 were zero. The lower forelimb placement scores observed in the neflamapimod-treated animals suggest a treatment effect of neflamapimod on recovery of somatosensory function following ischemic stroke.

\section{Study termination biomarker evaluation providing evidence for dose-}

\section{dependent increase in brain BDNF protein levels mediated by}

\section{neflamapimod treatment, and showing no significant effect on IL-1 $\beta$}

\section{production}

To examine the potential effect of neflamapimod on relevant biochemical pathways following ischemic stroke, IL-1 $\beta$ and BDNF protein levels in homogenates of brain tissue harvested from the right (tMCAO side) and left (non-affected) hemisphere were determined by ELISA.

Specifically, on Day 44, two days following the final dose of test article, brains were harvested from all animals and samples were processed after the brains were divided into left and right hemisphere. Samples from 18 animals in each treatment group $(1.5 \mathrm{mg} / \mathrm{kg}$ neflamapimod, $4.5 \mathrm{mg} / \mathrm{kg}$ neflamapimod and vehicle control) were analyzed.

Interestingly, IL-1 $\beta$ levels were variably increased in a subset of animals in each group in the injured right brain hemisphere (Table 3). IL-1 $\beta$ was undetectable (i.e. below LLOQ; IL-1 $\beta=0$ pg/ml) in the uninjured left brain hemisphere of all animals. The mean \pm standard error of the residual mean (SEM) IL-1 $\beta$ levels in the right hemisphere (after correction for an assay noise level of $5 \mathrm{pg} / \mathrm{ml}$ ) were numerically lower in each neflamapimod dose group $(14.6 \pm 5.1 \mathrm{pg} / \mathrm{ml}$ in the $1.5 \mathrm{mg} / \mathrm{kg}$ group 
and $15.2 \pm 6.7 \mathrm{pg} / \mathrm{ml}$ in the $4.5 \mathrm{mg} / \mathrm{kg}$ group) compared to the vehicle group $(29.3 \pm 12.4 \mathrm{pg} / \mathrm{ml})$, but there was no statistically significant difference between these groups. Thus, no clear neflamapimod effect on IL-1 $\beta$ could be detected on Day 44 post stroke.

Table 3. IL-1 $(\mathrm{pg} / \mathrm{ml})$ levels in the injured right brain hemisphere on Day 44 post stroke.

\begin{tabular}{|c|c|c|c|c|c|}
\hline \multicolumn{2}{|l|}{ Vehicle } & \multicolumn{2}{|c|}{$1.5 \mathrm{mg} / \mathrm{kg}$ NFMD } & \multicolumn{2}{|c|}{$4.5 \mathrm{mg} / \mathrm{kg}$ NFMD } \\
\hline $\begin{array}{l}\text { Rat } \\
\text { Number }\end{array}$ & 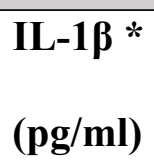 & $\begin{array}{l}\text { Rat } \\
\text { Number }\end{array}$ & 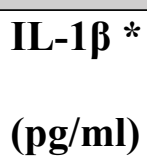 & $\begin{array}{l}\text { Rat } \\
\text { Number }\end{array}$ & $\begin{array}{l}\text { IL-1 } * \\
(\mathrm{pg} / \mathrm{ml})\end{array}$ \\
\hline 2 & 17.2 & 1 & 15.2 & 9 & 0 \\
\hline 8 & 37.5 & 4 & 0 & 12 & 0 \\
\hline 16 & 29.3 & 5 & 13.2 & 14 & 65.0 \\
\hline 20 & 4.4 & 18 & 62.5 & 15 & 96.8 \\
\hline 28 & 0 & 23 & 0 & 22 & 0 \\
\hline 29 & 12.8 & 27 & 19.9 & 24 & 0 \\
\hline 33 & 183.5 & 31 & 0 & 25 & 25.1 \\
\hline 38 & 0 & 40 & 0 & 26 & 0 \\
\hline 43 & 146.1 & 41 & 0 & 32 & 50.8 \\
\hline 45 & 50.8 & 42 & 0 & 34 & 0 \\
\hline 51 & 45.7 & 44 & 47.8 & 36 & 0 \\
\hline 52 & 0 & 46 & 33.1 & 39 & 0 \\
\hline 54 & 0 & 50 & 1.3 & 47 & 1.3 \\
\hline 56 & 0 & 53 & 0 & 57 & 34.6 \\
\hline 62 & 0 & 55 & 0 & 58 & 0 \\
\hline
\end{tabular}


PAGE 23

\begin{tabular}{|l|l|l|l|l|l|}
\hline 64 & 0 & 60 & 61.0 & 59 & 0 \\
\hline 69 & 0 & 73 & 0 & 75 & 0 \\
\hline 77 & 0 & 74 & 8.9 & 76 & 0 \\
\hline Mean & $\mathbf{2 9 . 3}$ & Mean & $\mathbf{1 4 . 6}$ & Mean & $\mathbf{1 5 . 2}$ \\
\hline SD & $\mathbf{5 2 . 6}$ & SD & $\mathbf{2 1 . 8}$ & SD & $\mathbf{2 8 . 5}$ \\
\hline SEM & $\mathbf{1 2 . 4}$ & SEM & $\mathbf{5 . 1}$ & SEM & $\mathbf{6 . 7}$ \\
\hline
\end{tabular}

* IL-1 $\beta$ values after subtraction of the LLOQ value of $5 \mathrm{pg} / \mathrm{ml}$.

Additionally, BDNF protein was detectable in both brain hemispheres of all animals on Day 44 post stroke as shown in Fig 3. Within each brain hemisphere there was a significant dose-related effect of neflamapimod for increasing BDNF protein levels (Jonckheere-Terpstra test: $P<0.01$ and $P<0.05$ for the left (Fig 3A) and right (Fig 3B) hemisphere, respectively). In addition, in the left hemisphere (non-affected side), the mean \pm SEM BDNF levels were statistically significantly higher in the $4.5 \mathrm{mg} / \mathrm{kg}$ group $(2,762 \pm 271.6 \mathrm{pg} / \mathrm{ml})$ than in the vehicle group $(2,010 \pm 149.7 ; P<0.05)$. The left hemisphere mean \pm SEM BDNF levels in the $1.5 \mathrm{mg} / \mathrm{kg}$ group $(2,213 \pm 136.0 \mathrm{pg} / \mathrm{ml})$ were intermediate to those in the vehicle and the $4.5 \mathrm{mg} / \mathrm{kg}$ group, although not statistically significantly different from the vehicle group when the $1.5 \mathrm{mg} / \mathrm{kg}$ and vehicle groups were compared directly.

\section{Fig 3. Dose-related neflamapimod (NFMD) subacute six-week treatment effects on rat brain}

BDNF levels analyzed on Day 44 post tMCAO. Brain homogenate samples from 18 animals in each treatment group (vehicle, $1.5 \mathrm{mg} / \mathrm{kg}$ neflamapimod, and $4.5 \mathrm{mg} / \mathrm{kg}$ neflamapimod) were prepared two days after the six-week treatment period and analyzed by BDNF ELISA. Data are presented as a technical data plot where the result for each individual animal is represented as one 


\section{PAGE 24}

dot, the horizontal long line represents the mean of all values within a group, and the upper and lower range of the SEM are presented. (A) Right hemisphere (tMCAO): A significant neflamapimod dose trend by the Jonckheere-Terpstra test for higher BDNF levels was observed $(P<0.05)$ for across the three groups. (B) Left hemisphere (non-injured side): The Jonckheere-Terpstra test indicated a significant neflamapimod dose trend for higher BDNF levels in the left hemisphere $(P<0.01)$. The mean \pm SEM BDNF level was also statistically significantly higher in the $4.5 \mathrm{mg} / \mathrm{kg}$ neflamapimod group when compared with the vehicle control group $(P<0.05)$.

\section{Discussion}

The results for the daily general health assessments, the weekly body weight measurements, the neurologic recovery assessments on Day 2, at Week 4 and at Week 6 post stroke, and the terminal biomarker measurements on Day 44 in this rat tMCAO study taken together led to the following seven key observations for evaluation of delayed and prolonged neflamapimod treatment. (1) Neflamapimod treatment dose-dependently and continually improved neurologic and behavioral outcomes assessed by mNSS and specific measures of sensory and motor function post stroke (Fig 1 and Fig 2). (2) Neflamapimod dose-dependently increased terminal brain BDNF protein as a neurogenic factor biomarker measure for beneficial effects on synaptic plasticity (Fig 3). (3) Neflamapimod and vehicle treatments were generally well-tolerated throughout the study, providing no treatment-related adverse clinical signs. (4) There was an average body weight gain of $27 \%$ in all treatment groups throughout the six-week study period (Table 2) and there was no difference observed in the general health of the rats that would have contributed to any neflamapimod versus vehicle treatment-mediated functional recovery. (5) Slight, albeit statistically significant spontaneous recovery over time was also observed in the vehicle control group (an average $\sim 19 \%$ decrease in 


\section{PAGE 25}

mNSS value within six weeks; Fig 1) implying that neflamapimod in vivo efficacy may enhance a biologic process, like neural or synaptic plasticity, that is already active. (6) Generally, the functional recovery was slow and steady in all neflamapimod- or vehicle-treated animals throughout the prolonged study period (Fig 1 and Fig 2). (7) There were signs for unresolved IL-1 $\beta$-mediated chronic inflammation in the injured brain hemisphere of a subset of animals, however, no clear neflamapimod effect on IL-1 $\beta$ production was apparent at study termination (Table 3). Taken together, the results for this research study provide evidence that there is an additional clinical opportunity for $\mathrm{p} 38 \alpha$ inhibitors in ischemic stroke since neflamapimod widened the window to treatment initiation to 48 hours post stroke in a rat tMCAO model and promoted recovery.

The most important finding of this study is that the delayed and prolonged treatment with neflamapimod resulted in dose-related neurologic and behavioral improvements versus vehicle control, when this $\mathrm{p} 38 \alpha$ inhibitor was administered orally to rats with tMCAO in an experimental paradigm of promoting synaptic plasticity and functional recovery during the subacute phase of ischemic stroke. To this end, neflamapimod treatment started at 48 hours after reperfusion at a time when the acute stroke phase was considered complete, since this represents a time point that is outside the previously characterized neuroprotection window of less than 24-hours post tMCAOinduced stroke for a p38 $\alpha$ inhibitor [25]. In the most relevant (based experimental stroke model and rat strain) previously published study, the p38 MAPK inhibitor SB203580 was administered via intracerebroventricular injection 30 minutes pre-tMCAO, or at 6,12 or 24 hours post-tMCAO to Sprague Dawley rats and demonstrated a decrease in infarct volume that was associated with antiinflammatory effects in the injured brain and improved recovery from neurologic deficit measured on Day 2 post stroke for all the treatment initiations up to 12 hours post-tMCAO, whereas no beneficial effect was observed for the treatment initiation at 24 hours post tMCAO [25]. The 


\section{PAGE 26}

observation that a 12-hour time window for the start of $\mathrm{p} 38 \alpha$ inhibition, but not a 24-hour window allowed for neuroprotective effects in the rat tMCAO-induced stroke model supports the selection of 48-hours as the time point for initiation of subacute phase neflamapimod dosing in the current study, since the results by Piao et al. [25] indicated that it is too late a time point for a p38 $\alpha$ inhibitor in this type of model to lead to reduction of neuronal loss as a mechanism for impacting functional outcome.

This neflamapimod treatment study is clearly different from all previously reported studies of other small molecule p38 MAPK inhibitors in various experimental stroke models [25, 28-32] both in terms of the delay in treatment initiation to 48 hours post reperfusion and the six-week prolonged dosing duration and overall treatment study focus. All previously reported studies of p38 MAPK inhibitors that most potently inhibit the p38 $\alpha$ isoform, including SB203580 [25], SB239063 [28, 29], RWJ67657 [31], or VCP979 [32], involved compound administration before or soon after the experimental stroke during the acute phase, with treatment initiations at different time points, ranging from as early as 1 hour pre stroke to as late as 24 hours post stroke. Additionally, all prior p38 $\alpha$ inhibitor studies had a focus on demonstrating neuroprotective effects that resulted in significant reduction of infarct volume (assessed as early as 6 hours post stroke) and subsequent improvements of neurological defects assessed at 24 hours [28, 29], 2 days [25], 7 days [32] or 14 days post stroke [31]. The two most recent p38 $\alpha$ inhibitor studies [31, 32] were also the two longest ones prior to the present study. Interestingly, they did demonstrate the validity of mNSS [51] as a readout for functional recovery attributable to neuroprotection via $\mathrm{p} 38 \alpha$ inhibition, that was measurable during the subacute phase after photothrombotic ischemic stroke in mice at one and two weeks, respectively $[31,32]$. The results of this neflamapimod study extend these positive mNSS effect observations for a $\mathrm{p} 38 \alpha$ inhibitor to a rat tMCAO model. In contrast to all previous $\mathrm{p} 38 \alpha$ 
inhibitor studies, the actual size of the acute cerebral infarct was not measured in this neflamapimod study since the main objective was to evaluate the compound as a therapy for post stroke functional recovery, not as a neuroprotection therapy, and the delay in treatment initiation to 48 hours post stroke was expected to prevent a neuroprotection immediate impact of the compound on infarct size based on the previously published p38 $\alpha$ inhibitor results [25]. Therefore, infarct volume measurement was not relevant for evaluation of neflamapimod due its late application as experimental stroke treatment. Instead, evaluation of mNSS [51] and additional functional tests (stepping test, body swing test, forelimb placement test) to assess the sensory motor deficit after stroke together with BDNF protein biomarker effects were deemed sufficient to evaluate and demonstrate the beneficial in vivo efficacy of neflamapimod.

Since the present study represents a first both for initiating the dosing of a potent and selective p38 $\alpha$ inhibitor as late as 48 hours in the subacute phase after rodent tMCAO-induced stroke, as well as for dosing this type of a small molecule for a prolonged time period of six weeks, it is a challenge to find directly supportive subacute or chronic stroke phase studies. It is reassuring that the functional recovery demonstrated in this study is consistent with a report by Umezawa et al. [52] who demonstrated that selective inhibition of $\mathrm{p} 38 \alpha$ by SB239063 improved locomotor recovery after SCI in mice. Although in a different disease context, this study may be most relevant since Umezawa et al. [52] also utilized a genetic approach (i.e. a heterozygous knockout of MAPK14) to identify the p38 $\alpha$ specificity of the effect. Generally, small molecule treatments in the rodent cerebral ischemia model start before the 48-hour time point after reperfusion, as already discussed for all the previously reported p38 $\alpha$ inhibitor studies [25, 28-32]. Those studies are not inconsistent with this neflamapimod study, only different in that the neurologic recovery effects therein are clearly associated with a primary acute phase neuroprotective effect of the p38 $\alpha$ inhibitors [25, 28- 


\section{PAGE 28}

32]. A review of the experimental stroke literature, however, revealed that the results of all these studies are in conflict with several reports that have associated elevated p38 MAPK activity, including p38 $\alpha$ activity, with a neuroprotective role during and after stroke, either in the acute or subacute phase [53-59]. One caveat is that several of these differing studies used the small molecule SB203580 at a relatively high concentration and did not address the possibility of blocking p38 MAPK isoforms other than $\mathrm{p} 38 \alpha$, such as p38 $\beta$, or other kinases (e.g. GAK, RIPK2, NLK, JNK3, CSNK1D and others) with this less potent and much less selective p38 $\alpha$ inhibitor tool compound [60]. As an example, SB203850 through inhibiting casein kinase I $\delta$ and $\varepsilon$ blocks WNT-stimulated $\beta$ catenin signaling $[61,62]$, which might account for compound effects on neurogenesis, therefore, render claims for p38 attributed activities invalid [56]. While it is likely that the role of p38 $\alpha$ depends on context, which may include the model species or strain, the type of stroke model, the temporal characteristics, the local concentration of the target protein and its substrates, the target cell type, the presence or absence of pathway regulators, and possibly other factors, it appears that additional experimental analyses involving low concentrations of neflamapimod would have been useful to clarify the role of $\mathrm{p} 38 \alpha$ in some of these conflicting research studies. Based on its low selectivity entropy score in addition to suitable physical, biochemical and cellular properties, neflamapimod has been recommended as the small molecule compound to utilize in experimental studies that have the objective of understanding the biologic effects of inhibiting $\mathrm{p} 38 \alpha$ kinase activity specifically [63].

The rat tMCAO model used in this study is one of the most frequently used in vivo models in stroke research, partially because the procedure used for stroke induction causes reproducible infarcts in the middle cerebral artery $[33,64]$. The tMCAO procedure was performed under highly standardized and well-controlled conditions [65] by well-trained and experienced scientists to reduce 


\section{PAGE 29}

the inter-animal variability of infarct size to a minimum, which is demonstrated by consistently high mNSS values determined on Day 2. The mean \pm SD mNSS value was $14.0 \pm 1.4$ for the 67 rats prior to their randomized assignment into the three different treatment groups. This a relatively tight value and an indication of a robust stroke since it represents a tremendous increase from the mNSS value of 0 reflecting the normal, healthy pre-tMCAO status prior to Day 1 .

Even though the technical procedures used for this experimental model have been designed to have a low mortality associated with the robust stroke induction, mortality of up to $25 \%$ within 24 hours after stroke onset is quite common [66-68] and mortality has been reported to increase up to $33 \%$ in the following days due to edema $[69,70]$. In this tMCAO study, 9 out of 76 animals $(\sim 12 \%)$ were lost during the first 24 hours post stroke, 4 more until 48 hours post stroke, and 3 more during the first two weeks of treatment, resulting in $\sim 21 \%$ mortality within the first 16 days after tMCAO. These results are well within the range of the expected mortality results for rodent stroke models [66-70]. Intriguingly, the mortality results in these experimental models also somewhat mirror human data where mortality within 28 days of stroke has been described to range up to $28 \%$ [ 71 , 72].

Oral neflamapimod was dosed at 1.5 and $4.5 \mathrm{mg} / \mathrm{kg}$ administered in 1\% Pluronic F108 vehicle twice daily on 5.5 days per week for six weeks in the present rat tMCAO study. These two neflamapimod dose levels in this vehicle were selected because their twice daily administration for three weeks was previously demonstrated to be pharmacologically active in aged rats with cognitive deficits attributed to chronic inflammation-induced, IL-1 $\beta$-mediated impairment of synaptic plasticity [47]. The results obtained in this tMCAO study corroborate that these two neflamapimod dose levels are pharmacologically active in rats. Unlike the aged rat study, in which the $4.5 \mathrm{mg} / \mathrm{kg}$ dose did not lead to better cognitive results compared to the $1.5 \mathrm{mg} / \mathrm{kg}$ dose [47], the beneficial 
effects by neflamapimod resulting in neurologic and behavioral improvements after stroke increased in a dose-related manner and enhanced the slight spontaneous recovery observed in the vehicle control group. Similar to the aged rat study [47], the beneficial effects of neflamapimod in this experimental stroke study do not appear to be due to inhibition of IL-1 $\beta$ production. There was no statistically significant decrease in the terminal IL-1 $\beta$ brain levels at either neflamapimod dose level compared to vehicle control.

The IL- $1 \beta$ results of the present rat tMCAO study are limited to one time point only and show that a residual elevation of IL-1 $\beta$ on the stroked side of the brain, but not on the unaffected side, was measurable in $50 \%$ of animals in the vehicle- and low-dose neflamapimod-treated group as well as in $30 \%$ of the animals in the high-dose neflamapimod-treated group at the end of the study. This together with the observed beneficial neurologic effects by neflamapimod suggests that chronic inflammation may play a role in this rat tMCAO stroke model. This is a finding that supplements previously reported IL-1 $\beta$ results in this type of model $[36,73,74]$. Although this single time point analysis appears to exclude a neflamapimod effect on IL-1 $\beta$ production, it does not necessarily preclude a neflamapimod effect on IL-1 $\beta$ signaling, even though a correlative relationship of the Day 44 IL-1 $\beta$ levels with the six-week mNSS values or with their Day 44 BDNF levels was not found. The present study was not intended to address the exact mechanism of action of neflamapimod and the IL-1 $\beta$ and BDNF data are very limited.

IL-1 $\beta$ is a pleiotropic cytokine that is known to be upregulated acutely after ischemic stroke in the rat and has been demonstrated to be a contributor to pathology via p38 $\alpha$ activity [25].

Previously published temporal profiles of IL-1 $\beta$ mRNA or protein in experimental stroke, which are generally limited to a time period up to 5 days post stroke, have demonstrated that IL- $1 \beta$ is increasing shortly after stroke, usually peaking within the first 24 hours during the acute phase, and 
then declining towards baseline levels or still remaining elevated $[36,73,74]$. While it is clear that acutely elevated levels of IL-1 $\beta$ exert potent pro-inflammatory functions and play a role in initiation of tMCAO-induced damage $[75,76]$, less is known about IL-1 $\beta$ dynamics as a result of stroke damage, or about what goes on mechanistically during the subacute stroke phase [37]. The results of the present study are in line with studies that demonstrate that subacute phase IL-1 $\beta$ continues to potentiate neuronal injury in experimental stroke [37]. They run counter to other studies that argue that subacute/chronic IL-1 $\beta$ after experimental stroke serves to halt or repair injury [37]. It is possible that these controversial results may be context-dependent (e.g. species/strain, age of animals, experimental stroke model, size of stroke damage, involved cell types) and additional research will be needed to sort out the relevance of the different outcomes and the mechanisms for IL-1 $\beta$-mediated responses [37]. It is clear that modeling inflammation and immunity in experimental models of stroke is challenging even though the sensitivity of rodents to focal cerebral ischemia as well as their physiology and pathophysiology are sufficiently similar to humans to make them a highly relevant model organism [77, 78].

Terminal measurements of brain BDNF protein demonstrated that six-week neflamapimod treatment resulted in dose-dependent increases of this neurotrophic factor in both the injured and uninjured hemisphere. BDNF is a key regulator of plasticity both in the healthy and injured brain which has been recognized as a key regulator of rehabilitation- and activity-induced functional and motor recovery, respectively, after stroke [79-81]. BDNF is reported to have a critical role in promoting recovery after stroke as a crucial signaling molecule that mediates adaptive brain plasticity [35, 82-85]. Increased BDNF levels in perilesional areas have been observed with interventions that improve functional recovery post stroke [86-88]. Conversely, attenuation of brain BDNF levels or effects following cerebral ischemia results in reduced neuroplastic changes or 
PAGE 32

decreased recovery of function, both in spontaneous and in rehabilitation-induced recovery scenarios $[84,89,90]$. Since regenerative roles have been attributed to BDNF in preclinical models of stroke [84, 91-94], upregulation of BDNF may be a plausible contributor to the neflamapimod-induced functional recovery observed after the ischemic stroke.

How BDNF elevation is linked to $\mathrm{p} 38 \alpha$ inhibition by neflamapimod in this experimental stroke model will need to be determined in a follow-up mechanistic study. Our favored hypothesis is that the BDNF levels might be interpreted as a marker of a more general effect on IL-1 $\beta$ signaling [42] that could result from $\mathrm{p} 38 \alpha$ inhibition $[41,43]$. In such a hypothetical mechanistic model, the observation of inhibition of IL-1 $\beta$ signaling without impacting IL-1 $\beta$ production would be consistent with previously observed aged rat results demonstrating that neflamapimod is more potent at inhibiting IL-1 $\beta$ signaling than at inhibiting IL-1 $\beta$ production [47]. While this stroke study was not intended to address the exact mechanism of action of neflamapimod, the observation that BDNF protein was increased in both brain hemispheres at study termination is nevertheless supporting the underlying hypothesis that functional recovery was associated with enhancing synaptic plasticity.

Taking into account the design of the study that precludes an effect through neuroprotection, the absence of an anti-inflammatory effect, and the possibility of an effect on IL-1 $\beta$ signaling, combined with the scientific literature regarding p38 MAPK-mediated deleterious effects of IL-1 $\beta$ on synaptic plasticity $[43,95]$, the results herein imply a model in which IL-1 $\beta$ would limit functional recovery after stroke via p38 $\alpha$-mediated impairment of neural and synaptic plasticity. The results in this neflamapimod-mediated stroke recovery study are also consistent with studies in other disease contexts in which activation of p38 MAPK, particularly the alpha isoform, is associated with impaired synaptic plasticity $[18,96]$. Further, the non-clinical to clinical translational potential with neflamapimod as a p38 $\alpha$ inhibitor is that, similar to the observed slight spontaneous recovery 
observed in the vehicle control group, neural and synaptic plasticity has been argued to be active in humans and at least partially effective in recovery after stroke [97]. Enhancing a biologic process like neural or synaptic plasticity, that is already active, rather than targeting a process that may not represent an intrinsic recovery pathway, such as neurogenesis, should at least theoretically be more likely to have a clinical effect. For this reason, the opportunity of translational success with p $38 \alpha$ inhibition to promote recovery following stroke by enhancing plasticity would be expected to be higher than for neuroprotection.

This research study can also be considered as a non-clinical development study that assesses the in vivo efficacy of neflamapimod, a highly selective p38 $\alpha$ inhibitor, in the commonly used rat tMCAO model of stroke $[33,64]$. Even though the promptly restored blood flow in this experimental model is different from the pathophysiology in spontaneous human stroke, the rat tMCAO model closely mimics the therapeutic situation of mechanical thrombectomy which is expected to be increasingly applied to patients [33]. This suggests that this rat ischemia model may have clinical relevance [33].

Potentially the most important consideration for clinical translation when targeting recovery from stroke is the practical consideration of time window for therapeutic intervention. The narrow time window after onset of ischemia that is required of neuroprotective approaches has posed an insurmountable challenge for clinical development [98]. Stroke patients do not often present within the required first few hours and, even within the initial 24 hours post-stroke, the clinical presentation does not allow one to assess the true size and severity of the stroke. Therefore, any clinical study that starts treatment of patients within the first day of the stroke has a highly variable and heterogeneous patient population and thus requires a large sample size to demonstrate clinical effects. These restrictions preclude the ability to demonstrate clinical proof-of-concept in phase 2 clinical testing. 


\section{PAGE 34}

In contrast, a time window of 24 to 48 hours after stroke allows patients' clinical course to have stabilized and allows time for a clinical exam and a diffusion-MRI scan to precisely determine the location and extent of the stroke before starting treatment. In addition, a reasonable prognostic indication of the extent of recovery that a patient will attain with or without intervention can be assessed. As a result, starting treatment in a clinical study at 48 hours or later after stroke allows for the inclusion of more homogenous sub-populations of patients and increases statistical power with fewer subjects; therefore, definitive clinical-proof-of-concept could potentially be demonstrated within a phase 2 clinical study.

Despite the observation of robust in vivo efficacy of neflamapimod, there are some limitations to this research stroke study, since only one treatment duration was chosen, and functional outcomes were measured at the Week 4 and Week 6 timepoints after the stroke. It cannot be predicted whether longer neflamapimod treatment duration with additional analyses would have led to further mNSS improvement and continued recovery during the chronic phase, and the exact timing of the onset of neflamapimod action on functional recovery was not determined. Neflamapimod treatment was initiated at 48 hours after reperfusion, at a time point that was considered subacute post stroke in light of the prior negative experiences with other $\mathrm{p} 38 \alpha$ inhibitors and numerous neuroprotective agents when they were administered at 24 hours or more post-stroke, which implies an exceedingly low likelihood that neuroprotection plays a role in promoting functional recovery, but without accompanying histologic analyses an effect of neflamapimod on neuronal loss cannot be formally excluded. Further, while we believe that it is highly probable that the functional recovery mediated by neflamapimod was due to enhancement of plasticity mechanisms, in the absence of technologies that can measure synaptic function in vivo in real-time, there is no means to directly verify that assumption. Similarly, while the known activity of IL- $1 \beta$ to 


\section{PAGE 35}

impair synaptic plasticity via $\mathrm{p} 38 \alpha$ implies that inhibition IL-1 $\beta$ activity is a major contributor to the neflamapimod clinical activity, there is no means to directly confirm that link in vivo, or to exclude other potential mechanisms. Finally, in the current study, young (3-months old) male animals were utilized. Although neural and synaptic plasticity recovery functions appear to be active in aged animals and are also at least partially preserved in elderly patients [99], in order to improve clinical translation, a replication and extension of this study to include females, aged animal and animals with co-morbidities may be required since gender, age and reduced health condition (e.g. illnesses, diseases, disorders, health problems) may affect development of ischemic damage and resulting behavioral deficits in patients [100].

The clinical science translational opportunity with neflamapimod is that the compound has already been studied in early Alzheimer's disease patients at the human dose equivalents of 1.5 and $4.5 \mathrm{mg} / \mathrm{kg}$ in the rat [46]. These doses were well-tolerated, achieved target cerebral spinal fluid (CSF) drug levels consistent with robust blood-brain-barrier penetration, and demonstrated target activity as assessed by imaging and CSF biomarkers. Thus, neflamapimod provides the opportunity of evaluation in the clinic to address whether its $\mathrm{p} 38 \alpha$ inhibition will promote recovery after ischemic stroke, and such a study expected to provide insights into its potential to enhance neural and synaptic plasticity mechanisms in humans.

\section{Conclusions}

Here prolonged, six-week oral administration of the p38 $\alpha$ inhibitor neflamapimod, with treatment initiation starting at 48 hours post reperfusion that is outside the previously characterized neuroprotection window for $\mathrm{p} 38 \alpha$ inhibitors, resulted in dose-related significant neurologic recovery and improvement of motor and sensory functions measured at four and six weeks post stroke. 
PAGE 36

Additionally, dose-related increases of the neurogenic factor BDNF in the brain as a potential biomarker for neflamapimod effects on synaptic plasticity were observed at termination of the study on Day 44. Thus, neflamapimod use offers the possibility of widening the window for treatment initiation post stroke and promoting recovery after a completed stroke. Since it is also known to counteract cognitive decline in aged rats a well as Alzheimer patients, neflamapimod is clinically developable and especially appealing as potential stroke therapy.

\section{Acknowledgments}

The authors would like to acknowledge the help of all the people involved in this project, in particular Dr. Itschak Lamensdorf at Pharmaseed, who provided inspiration and interpretive perspective on the results. Additionally, Molly Opferman as consulting medical writer provided technical writing assistance in preparing the manuscript.

\section{References}

1. Disease GBD, Injury I, Prevalence C. Global, regional, and national incidence, prevalence, and years lived with disability for 354 diseases and injuries for 195 countries and territories, 1990-2017: a systematic analysis for the Global Burden of Disease Study 2017. Lancet. 2018;392(10159):1789-858. Epub 2018/11/30. doi: 10.1016/S0140-6736(18)32279-7. PubMed PMID: 30496104; PubMed Central PMCID: PMCPMC6227754.

2. Rajsic S, Gothe H, Borba HH, Sroczynski G, Vujicic J, Toell T, et al. Economic burden of stroke: a systematic review on post-stroke care. Eur J Health Econ. 2019;20(1):107-34. Epub 2018/06/18. doi: 10.1007/s10198-018-0984-0. PubMed PMID: 29909569. 
3. Lipton P. Ischemic cell death in brain neurons. Physiol Rev. 1999;79(4):1431-568. Epub 1999/10/03. doi: 10.1152/physrev.1999.79.4.1431. PubMed PMID: 10508238.

4. Hacke W, Lichy C. Thrombolysis for acute stroke under antiplatelet therapy: safe enough to be beneficial? Nat Clin Pract Neurol. 2008;4(9):474-5. Epub 2008/07/31. doi: 10.1038/ncpneuro0867. PubMed PMID: 18665145.

5. Grotta JC, Hacke W. Stroke Neurologist's Perspective on the New Endovascular Trials. Stroke. 2015;46(6):1447-52. Epub 2015/05/07. doi: 10.1161/STROKEAHA.115.008384. PubMed PMID: 25944328.

6. Zhao LR, Willing A. Enhancing endogenous capacity to repair a stroke-damaged brain: An evolving field for stroke research. Prog Neurobiol. 2018;163-164:5-26. Epub 2018/02/25. doi: 10.1016/j.pneurobio.2018.01.004. PubMed PMID: 29476785; PubMed Central PMCID: PMCPMC6075953.

7. Cassidy JM, Cramer SC. Spontaneous and Therapeutic-Induced Mechanisms of Functional Recovery After Stroke. Transl Stroke Res. 2017;8(1):33-46. Epub 2016/04/26. doi: 10.1007/s12975-016-0467-5. PubMed PMID: 27109642; PubMed Central PMCID: PMCPMC5079852.

8. Cramer SC. Treatments to Promote Neural Repair after Stroke. J Stroke. 2018;20(1):57-70. Epub 2018/02/07. doi: 10.5853/jos.2017.02796. PubMed PMID: 29402069; PubMed Central PMCID: PMCPMC5836581.

9. Grupke S, Hall J, Dobbs M, Bix GJ, Fraser JF. Understanding history, and not repeating it. Neuroprotection for acute ischemic stroke: from review to preview. Clin Neurol Neurosurg. 2015;129:1-9. Epub 2014/12/17. doi: 10.1016/j.clineuro.2014.11.013. PubMed PMID: 25497127. 


\section{PAGE 38}

10. Karsy M, Brock A, Guan J, Taussky P, Kalani MY, Park MS. Neuroprotective strategies and the underlying molecular basis of cerebrovascular stroke. Neurosurg Focus. 2017;42(4):E3. Epub 2017/04/04. doi: 10.3171/2017.1.FOCUS16522. PubMed PMID: 28366066.

11. Xiong XY, Liu L, Yang QW. Refocusing Neuroprotection in Cerebral Reperfusion Era: New Challenges and Strategies. Front Neurol. 2018;9:249. Epub 2018/05/10. doi:

10.3389/fneur.2018.00249. PubMed PMID: 29740385; PubMed Central PMCID: PMCPMC5926527.

12. Venkat P, Shen Y, Chopp M, Chen J. Cell-based and pharmacological neurorestorative therapies for ischemic stroke. Neuropharmacology. 2018;134(Pt B):310-22. Epub 2017/09/05. doi: 10.1016/j.neuropharm.2017.08.036. PubMed PMID: 28867364; PubMed Central PMCID: PMCPMC5832535.

13. Chico LK, Van Eldik LJ, Watterson DM. Targeting protein kinases in central nervous system disorders. Nat Rev Drug Discov. 2009;8(11):892-909. Epub 2009/10/31. doi:

10.1038/nrd2999. PubMed PMID: 19876042; PubMed Central PMCID: PMCPMC2825114.

14. Lee JK, Kim NJ. Recent Advances in the Inhibition of p38 MAPK as a Potential Strategy for the Treatment of Alzheimer's Disease. Molecules. 2017;22(8). Epub 2017/08/03. doi:

10.3390/molecules22081287. PubMed PMID: 28767069; PubMed Central PMCID: PMCPMC6152076.

15. Han J, Lee JD, Bibbs L, Ulevitch RJ. A MAP kinase targeted by endotoxin and hyperosmolarity in mammalian cells. Science. 1994;265(5173):808-11. Epub 1994/08/05. doi: 10.1126/science.7914033. PubMed PMID: 7914033.

16. Lee JC, Laydon JT, McDonnell PC, Gallagher TF, Kumar S, Green D, et al. A protein kinase involved in the regulation of inflammatory cytokine biosynthesis. Nature. 
1994;372(6508):739-46. Epub 1994/12/22. doi: 10.1038/372739a0. PubMed PMID: 7997261.

17. Borders AS, de Almeida L, Van Eldik LJ, Watterson DM. The p38alpha mitogen-activated protein kinase as a central nervous system drug discovery target. BMC Neurosci. 2008;9 Suppl 2:S12. Epub 2009/01/06. doi: 10.1186/1471-2202-9-S2-S12. PubMed PMID: 19090985; PubMed Central PMCID: PMCPMC2604896.

18. Correa SA, Eales KL. The Role of p38 MAPK and Its Substrates in Neuronal Plasticity and Neurodegenerative Disease. J Signal Transduct. 2012;2012:649079. Epub 2012/07/14. doi: 10.1155/2012/649079. PubMed PMID: 22792454; PubMed Central PMCID: PMCPMC3389708.

19. Bachstetter AD, Van Eldik LJ. The p38 MAP Kinase Family as Regulators of Proinflammatory Cytokine Production in Degenerative Diseases of the CNS. Aging Dis. 2010;1(3):199-211. Epub 2010/12/01. PubMed PMID: 22720195; PubMed Central PMCID: PMCPMC3377763.

20. Lee SH, Park J, Che Y, Han PL, Lee JK. Constitutive activity and differential localization of p38alpha and p38beta MAPKs in adult mouse brain. J Neurosci Res. 2000;60(5):623-31. Epub 2000/05/23. doi: 10.1002/(SICI)1097-4547(20000601)60:5<623::AIDJNR7>3.0.CO;2-4. PubMed PMID: 10820433.

21. Ferrer I, Friguls B, Dalfo E, Planas AM. Early modifications in the expression of mitogenactivated protein kinase (MAPK/ERK), stress-activated kinases SAPK/JNK and p38, and their phosphorylated substrates following focal cerebral ischemia. Acta Neuropathol. 2003;105(5):425-37. Epub 2003/04/05. doi: 10.1007/s00401-002-0661-2. PubMed PMID: 12677442. 
22. Krupinski J, Slevin M, Marti E, Catena E, Rubio F, Gaffney J. Time-course phosphorylation of the mitogen activated protein (MAP) kinase group of signalling proteins and related molecules following middle cerebral artery occlusion (MCAO) in rats. Neuropathol Appl Neurobiol. 2003;29(2):144-58. Epub 2003/03/29. doi: 10.1046/j.1365-2990.2003.00454.x. PubMed PMID: 12662322.

23. Nozaki K, Nishimura M, Hashimoto N. Mitogen-activated protein kinases and cerebral ischemia. Mol Neurobiol. 2001;23(1):1-19. Epub 2001/10/20. doi: 10.1385/MN:23:1:01. PubMed PMID: 11642541.

24. Piao CS, Che Y, Han PL, Lee JK. Delayed and differential induction of p38 MAPK isoforms in microglia and astrocytes in the brain after transient global ischemia. Brain Res Mol Brain Res. 2002;107(2):137-44. Epub 2002/11/12. doi: 10.1016/s0169-328x(02)00456-4. PubMed PMID: 12425942.

25. Piao CS, Kim JB, Han PL, Lee JK. Administration of the p38 MAPK inhibitor SB203580 affords brain protection with a wide therapeutic window against focal ischemic insult. J Neurosci Res. 2003;73(4):537-44. Epub 2003/08/05. doi: 10.1002/jnr.10671. PubMed PMID: 12898538.

26. Mao G, Ren P, Wang G, Yan F, Zhang Y. MicroRNA-128-3p Protects Mouse Against Cerebral Ischemia Through Reducing p38alpha Mitogen-Activated Protein Kinase Activity. J Mol Neurosci. 2017;61(2):152-8. Epub 2016/12/03. doi: 10.1007/s12031-016-0871-z. PubMed PMID: 27905005.

27. Han D, Scott EL, Dong Y, Raz L, Wang R, Zhang Q. Attenuation of mitochondrial and nuclear p38alpha signaling: a novel mechanism of estrogen neuroprotection in cerebral 
ischemia. Mol Cell Endocrinol. 2015;400:21-31. Epub 2014/12/03. doi:

10.1016/j.mce.2014.11.010. PubMed PMID: 25462588.

28. Barone FC, Irving EA, Ray AM, Lee JC, Kassis S, Kumar S, et al. SB 239063, a secondgeneration p38 mitogen-activated protein kinase inhibitor, reduces brain injury and neurological deficits in cerebral focal ischemia. J Pharmacol Exp Ther. 2001;296(2):312-21. Epub 2001/02/13. PubMed PMID: 11160612.

29. Legos JJ, Erhardt JA, White RF, Lenhard SC, Chandra S, Parsons AA, et al. SB 239063, a novel p38 inhibitor, attenuates early neuronal injury following ischemia. Brain Res. 2001;892(1):70-7. Epub 2001/02/15. doi: 10.1016/s0006-8993(00)03228-5. PubMed PMID: 11172750.

30. Legos JJ, McLaughlin B, Skaper SD, Strijbos PJ, Parsons AA, Aizenman E, et al. The selective p38 inhibitor SB-239063 protects primary neurons from mild to moderate excitotoxic injury. Eur J Pharmacol. 2002;447(1):37-42. Epub 2002/07/11. doi: 10.1016/s0014-2999(02)01890-3. PubMed PMID: 12106800.

31. Chang D, Wang YC, Bai YY, Lu CQ, Xu TT, Zhu L, et al. Role of P38 MAPK on MMP Activity in Photothrombotic Stroke Mice as Measured using an Ultrafast MMP Activatable Probe. Sci Rep. 2015;5:16951. Epub 2015/11/20. doi: 10.1038/srep16951. PubMed PMID: 26581247; PubMed Central PMCID: PMCPMC4652271.

32. Cai Y, Lu C, Xu T, Ma Y, Min S, Scammells P, et al. Diffusion Tensor Imaging Evaluation of Axonal/White Matter Remodeling in a Mouse Model of Diabetic Stroke Treated with Novel p38 MAPK Inhibitor, VCP979. J Biomed Nanotechnol. 2018;14(3):585-93. Epub 2018/04/18. doi: 10.1166/jbn.2018.2522. PubMed PMID: 29663930. 
33. Sommer CJ. Ischemic stroke: experimental models and reality. Acta Neuropathol. 2017;133(2):245-61. Epub 2017/01/09. doi: 10.1007/s00401-017-1667-0. PubMed PMID: 28064357; PubMed Central PMCID: PMCPMC5250659.

34. Li H, Zhang N, Lin HY, Yu Y, Cai QY, Ma L, et al. Histological, cellular and behavioral assessments of stroke outcomes after photothrombosis-induced ischemia in adult mice. BMC Neurosci. 2014;15:58. Epub 2014/06/03. doi: 10.1186/1471-2202-15-58. PubMed PMID: 24886391; PubMed Central PMCID: PMCPMC4039545.

35. Berretta A, Tzeng YC, Clarkson AN. Post-stroke recovery: the role of activity-dependent release of brain-derived neurotrophic factor. Expert Rev Neurother. 2014;14(11):1335-44. Epub 2014/10/17. doi: 10.1586/14737175.2014.969242. PubMed PMID: 25319267.

36. Lambertsen KL, Biber K, Finsen B. Inflammatory cytokines in experimental and human stroke. J Cereb Blood Flow Metab. 2012;32(9):1677-98. Epub 2012/06/29. doi: 10.1038/jcbfm.2012.88. PubMed PMID: 22739623; PubMed Central PMCID: PMCPMC3434626.

37. Hewett SJ, Jackman NA, Claycomb RJ. Interleukin-1beta in Central Nervous System Injury and Repair. Eur J Neurodegener Dis. 2012;1(2):195-211. Epub 2012/08/01. PubMed PMID: 26082912; PubMed Central PMCID: PMCPMC4465544.

38. Sobowale OA, Parry-Jones AR, Smith CJ, Tyrrell PJ, Rothwell NJ, Allan SM. Interleukin-1 in Stroke: From Bench to Bedside. Stroke. 2016;47(8):2160-7. Epub 2016/03/05. doi:

10.1161/STROKEAHA.115.010001. PubMed PMID: 26931154.

39. Lambertsen KL, Finsen B, Clausen BH. Post-stroke inflammation-target or tool for therapy? Acta Neuropathol. 2019;137(5):693-714. Epub 2018/11/30. doi: 10.1007/s00401-018-1930z. PubMed PMID: 30483945; PubMed Central PMCID: PMCPMC6482288. 
40. Pozzi D, Menna E, Canzi A, Desiato G, Mantovani C, Matteoli M. The Communication Between the Immune and Nervous Systems: The Role of IL-1beta in Synaptopathies. Front Mol Neurosci. 2018;11:111. Epub 2018/04/21. doi: 10.3389/fnmol.2018.00111. PubMed PMID: 29674955; PubMed Central PMCID: PMCPMC5895746.

41. Song C, Zhang Y, Dong Y. Acute and subacute IL-1beta administrations differentially modulate neuroimmune and neurotrophic systems: possible implications for neuroprotection and neurodegeneration. J Neuroinflammation. 2013;10:59. Epub 2013/05/09. doi:

10.1186/1742-2094-10-59. PubMed PMID: 23651534; PubMed Central PMCID: PMCPMC3656796.

42. Patterson SL. Immune dysregulation and cognitive vulnerability in the aging brain: Interactions of microglia, IL-1beta, BDNF and synaptic plasticity. Neuropharmacology. 2015;96(Pt A):11-8. Epub 2015/01/01. doi: 10.1016/j.neuropharm.2014.12.020. PubMed PMID: 25549562; PubMed Central PMCID: PMCPMC4475415.

43. Tong L, Prieto GA, Kramar EA, Smith ED, Cribbs DH, Lynch G, et al. Brain-derived neurotrophic factor-dependent synaptic plasticity is suppressed by interleukin-1beta via p38 mitogen-activated protein kinase. J Neurosci. 2012;32(49):17714-24. Epub 2012/12/12. doi: 10.1523/JNEUROSCI.1253-12.2012. PubMed PMID: 23223292; PubMed Central PMCID: PMCPMC3687587.

44. Duffy JP, Harrington EM, Salituro FG, Cochran JE, Green J, Gao H, et al. The Discovery of VX-745: A Novel and Selective p38alpha Kinase Inhibitor. ACS Med Chem Lett. 2011;2(10):758-63. Epub 2011/10/13. doi: 10.1021/ml2001455. PubMed PMID: 24900264; PubMed Central PMCID: PMCPMC4018046. 
45. Alam J, Blackburn K, Patrick D. Neflamapimod: Clinical Phase 2b-Ready Oral Small Molecule Inhibitor of p38alpha to Reverse Synaptic Dysfunction in Early Alzheimer's Disease. J Prev Alzheimers Dis. 2017;4(4):273-8. Epub 2017/11/29. doi:

10.14283/jpad.2017.41. PubMed PMID: 29181493.

46. Scheltens P, Prins N, Lammertsma A, Yaqub M, Gouw A, Wink AM, et al. An exploratory clinical study of p38alpha kinase inhibition in Alzheimer's disease. Ann Clin Transl Neurol. 2018;5(4):464-73. Epub 2018/04/25. doi: 10.1002/acn3.549. PubMed PMID: 29687023; PubMed Central PMCID: PMCPMC5899915.

47. Alam JJ. Selective Brain-Targeted Antagonism of p38 MAPKalpha Reduces Hippocampal IL-1beta Levels and Improves Morris Water Maze Performance in Aged Rats. J Alzheimers Dis. 2015;48(1):219-27. Epub 2015/09/25. doi: 10.3233/JAD-150277. PubMed PMID: 26401942; PubMed Central PMCID: PMCPMC4923728.

48. Lynch MA. Age-related neuroinflammatory changes negatively impact on neuronal function. Front Aging Neurosci. 2010;1:6. Epub 2010/06/17. doi: 10.3389/neuro.24.006.2009. PubMed PMID: 20552057; PubMed Central PMCID: PMCPMC2874409.

49. Longa EZ, Weinstein PR, Carlson S, Cummins R. Reversible middle cerebral artery occlusion without craniectomy in rats. Stroke. 1989;20(1):84-91. Epub 1989/01/01. doi: 10.1161/01.str.20.1.84. PubMed PMID: 2643202.

50. Chen J, Li Y, Wang L, Zhang Z, Lu D, Lu M, et al. Therapeutic benefit of intravenous administration of bone marrow stromal cells after cerebral ischemia in rats. Stroke. 2001;32(4):1005-11. Epub 2001/04/03. doi: 10.1161/01.str.32.4.1005. PubMed PMID: 11283404. 


\section{PAGE 45}

51. Schaar KL, Brenneman MM, Savitz SI. Functional assessments in the rodent stroke model. Exp Transl Stroke Med. 2010;2(1):13. Epub 2010/07/21. doi: 10.1186/2040-7378-2-13. PubMed PMID: 20642841; PubMed Central PMCID: PMCPMC2915950.

52. Umezawa H, Naito Y, Tanaka K, Yoshioka K, Suzuki K, Sudo T, et al. Genetic and Pharmacological Inhibition of p38alpha Improves Locomotor Recovery after Spinal Cord Injury. Front Pharmacol. 2017;8:72. Epub 2017/03/07. doi: 10.3389/fphar.2017.00072. PubMed PMID: 28261102; PubMed Central PMCID: PMCPMC5313485.

53. Lennmyr F, Ericsson A, Gerwins P, Ahlstrom H, Terent A. Increased brain injury and vascular leakage after pretreatment with p38-inhibitor SB203580 in transient ischemia. Acta Neurol Scand. 2003;108(5):339-45. Epub 2003/11/18. doi: 10.1034/j.16000404.2003.00129.x. PubMed PMID: 14616304.

54. Pfeilschifter W, Czech B, Hoffmann BP, Sujak M, Kahles T, Steinmetz H, et al. Pyrrolidine dithiocarbamate activates p38 MAPK and protects brain endothelial cells from apoptosis: a mechanism for the protective effect in stroke? Neurochem Res. 2010;35(9):1391-401. Epub 2010/06/02. doi: 10.1007/s11064-010-0197-0. PubMed PMID: 20514517.

55. Cheng CY, Lin JG, Tang NY, Kao ST, Hsieh CL. Electroacupuncture at different frequencies $(5 \mathrm{~Hz}$ and $25 \mathrm{~Hz})$ ameliorates cerebral ischemia-reperfusion injury in rats: possible involvement of p38 MAPK-mediated anti-apoptotic signaling pathways. BMC Complement Altern Med. 2015;15:241. Epub 2015/07/19. doi: 10.1186/s12906-015-0752-y. PubMed PMID: 26187498; PubMed Central PMCID: PMCPMC4506591.

56. Lin Y, Zhang JC, Yao CY, Wu Y, Abdelgawad AF, Yao SL, et al. Critical role of astrocytic interleukin-17 A in post-stroke survival and neuronal differentiation of neural precursor cells in adult mice. Cell Death Dis. 2016;7(6):e2273. Epub 2016/06/24. doi: 


\section{PAGE 46}

10.1038/cddis.2015.284. PubMed PMID: 27336717; PubMed Central PMCID:

PMCPMC5143370.

57. Cheng CY, Tang NY, Kao ST, Hsieh CL. Ferulic Acid Administered at Various Time Points Protects against Cerebral Infarction by Activating p38 MAPK/p90RSK/CREB/Bcl-2 AntiApoptotic Signaling in the Subacute Phase of Cerebral Ischemia-Reperfusion Injury in Rats. PLoS One. 2016;11(5):e0155748. Epub 2016/05/18. doi: 10.1371/journal.pone.0155748. PubMed PMID: 27187745; PubMed Central PMCID: PMCPMC4871485.

58. Cheng CY, Ho TY, Hsiang CY, Tang NY, Hsieh CL, Kao ST, et al. Angelica sinensis Exerts Angiogenic and Anti-apoptotic Effects Against Cerebral Ischemia-Reperfusion Injury by Activating p38MAPK/HIF-1alpha/VEGF-A Signaling in Rats. Am J Chin Med. 2017;45(8):1683-708. Epub 2017/11/11. doi: 10.1142/S0192415X17500914. PubMed PMID: 29121798.

59. Cheng CY, Kao ST, Lee YC. Angelica sinensis extract protects against ischemia-reperfusion injury in the hippocampus by activating p38 MAPK-mediated p90RSK/p-Bad and p90RSK/CREB/BDNF signaling after transient global cerebral ischemia in rats. J Ethnopharmacol. 2020;252:112612. Epub 2020/01/29. doi: 10.1016/j.jep.2020.112612. PubMed PMID: 31988015.

60. Davis MI, Hunt JP, Herrgard S, Ciceri P, Wodicka LM, Pallares G, et al. Comprehensive analysis of kinase inhibitor selectivity. Nat Biotechnol. 2011;29(11):1046-51. Epub 2011/11/01. doi: 10.1038/nbt.1990. PubMed PMID: 22037378.

61. Shanware NP, Williams LM, Bowler MJ, Tibbetts RS. Non-specific in vivo inhibition of CK1 by the pyridinyl imidazole p38 inhibitors SB 203580 and SB 202190. BMB Rep. 
2009;42(3):142-7. Epub 2009/04/02. doi: 10.5483/bmbrep.2009.42.3.142. PubMed PMID: 19336000; PubMed Central PMCID: PMCPMC4412876.

62. Verkaar F, van der Doelen AA, Smits JF, Blankesteijn WM, Zaman GJ. Inhibition of Wnt/beta-catenin signaling by p38 MAP kinase inhibitors is explained by cross-reactivity with casein kinase Idelta/varepsilon. Chem Biol. 2011;18(4):485-94. Epub 2011/04/26. doi: 10.1016/j.chembiol.2011.01.015. PubMed PMID: 21513885.

63. Uitdehaag JC, Verkaar F, Alwan H, de Man J, Buijsman RC, Zaman GJ. A guide to picking the most selective kinase inhibitor tool compounds for pharmacological validation of drug targets. Br J Pharmacol. 2012;166(3):858-76. Epub 2012/01/19. doi: 10.1111/j.14765381.2012.01859.x. PubMed PMID: 22250956; PubMed Central PMCID: PMCPMC3417414.

64. Mizuma A, Yenari MA. Anti-Inflammatory Targets for the Treatment of Reperfusion Injury in Stroke. Front Neurol. 2017;8:467. Epub 2017/09/25. doi: 10.3389/fneur.2017.00467. PubMed PMID: 28936196; PubMed Central PMCID: PMCPMC5594066.

65. Krakovsky M, Polianski V, Nimrod A, Higazi A, Leker RR, Lamensdorf I. THR-18, a 18mer peptide derived from PAI-1, is neuroprotective and improves thrombolysis by tPA in rat stroke models. Neurol Res. 2011;33(9):983-90. Epub 2011/11/15. doi: 10.1179/1743132811Y.0000000018. PubMed PMID: 22081002.

66. Kuge Y, Minematsu K, Yamaguchi T, Miyake Y. Nylon monofilament for intraluminal middle cerebral artery occlusion in rats. Stroke. 1995;26(9):1655-7; discussion 8. Epub 1995/09/01. doi: 10.1161/01.str.26.9.1655. PubMed PMID: 7660413.

67. Neumann-Haefelin T, Kastrup A, de Crespigny A, Yenari MA, Ringer T, Sun GH, et al. Serial MRI after transient focal cerebral ischemia in rats: dynamics of tissue injury, blood- 
brain barrier damage, and edema formation. Stroke. 2000;31(8):1965-72; discussion 72-3. Epub 2000/08/06. doi: 10.1161/01.str.31.8.1965. PubMed PMID: 10926965.

68. Henninger N, Sicard KM, Schmidt KF, Bardutzky J, Fisher M. Comparison of ischemic lesion evolution in embolic versus mechanical middle cerebral artery occlusion in Sprague Dawley rats using diffusion and perfusion imaging. Stroke. 2006;37(5):1283-7. Epub 2006/03/25. doi: 10.1161/01.STR.0000217223.72193.98. PubMed PMID: 16556883.

69. Aspey BS, Cohen S, Patel Y, Terruli M, Harrison MJ. Middle cerebral artery occlusion in the rat: consistent protocol for a model of stroke. Neuropathol Appl Neurobiol. 1998;24(6):48797. Epub 1999/01/15. doi: 10.1046/j.1365-2990.1998.00146.x. PubMed PMID: 9888159.

70. Modo M, Stroemer RP, Tang E, Veizovic T, Sowniski P, Hodges H. Neurological sequelae and long-term behavioural assessment of rats with transient middle cerebral artery occlusion. J Neurosci Methods. 2000;104(1):99-109. Epub 2001/02/13. doi: 10.1016/s01650270(00)00329-0. PubMed PMID: 11163416.

71. Matsumoto N, Whisnant JP, Kurland LT, Okazaki H. Natural history of stroke in Rochester, Minnesota, 1955 through 1969: an extension of a previous study, 1945 through 1954. Stroke. 1973;4(1):20-9. Epub 1973/01/01. doi: 10.1161/01.str.4.1.20. PubMed PMID: 4685804.

72. Bronnum-Hansen H, Davidsen M, Thorvaldsen P, Danish MSG. Long-term survival and causes of death after stroke. Stroke. 2001;32(9):2131-6. Epub 2001/09/08. doi: 10.1161/hs0901.094253. PubMed PMID: 11546907.

73. Wang X, Yue TL, Barone FC, White RF, Gagnon RC, Feuerstein GZ. Concomitant cortical expression of TNF-alpha and IL-1 beta mRNAs follows early response gene expression in transient focal ischemia. Mol Chem Neuropathol. 1994;23(2-3):103-14. Epub 1994/10/01. doi: 10.1007/bf02815404. PubMed PMID: 7702701. 
74. Berti R, Williams AJ, Moffett JR, Hale SL, Velarde LC, Elliott PJ, et al. Quantitative realtime RT-PCR analysis of inflammatory gene expression associated with ischemiareperfusion brain injury. J Cereb Blood Flow Metab. 2002;22(9):1068-79. Epub 2002/09/10. doi: 10.1097/00004647-200209000-00004. PubMed PMID: 12218412.

75. Liu T, McDonnell PC, Young PR, White RF, Siren AL, Hallenbeck JM, et al. Interleukin-1 beta mRNA expression in ischemic rat cortex. Stroke. 1993;24(11):1746-50; discussion 50-1. Epub 1993/11/01. doi: 10.1161/01.str.24.11.1746. PubMed PMID: 8236352.

76. Murray KN, Parry-Jones AR, Allan SM. Interleukin-1 and acute brain injury. Front Cell Neurosci. 2015;9:18. Epub 2015/02/24. doi: 10.3389/fncel.2015.00018. PubMed PMID: 25705177; PubMed Central PMCID: PMCPMC4319479.

77. Dirnagl U. Modeling immunity and inflammation in stroke: can mice be trusted? Stroke. 2014;45(9):e177-8. Epub 2014/07/26. doi: 10.1161/STROKEAHA.114.005640. PubMed PMID: 25061077.

78. Dirnagl U, Endres M. Found in translation: preclinical stroke research predicts human pathophysiology, clinical phenotypes, and therapeutic outcomes. Stroke. 2014;45(5):1510-8. Epub 2014/03/22. doi: 10.1161/STROKEAHA.113.004075. PubMed PMID: 24652307.

79. Mang CS, Campbell KL, Ross CJ, Boyd LA. Promoting neuroplasticity for motor rehabilitation after stroke: considering the effects of aerobic exercise and genetic variation on brain-derived neurotrophic factor. Phys Ther. 2013;93(12):1707-16. Epub 2013/08/03. doi: 10.2522/ptj.20130053. PubMed PMID: 23907078; PubMed Central PMCID: PMCPMC3870490.

80. Crozier J, Roig M, Eng JJ, MacKay-Lyons M, Fung J, Ploughman M, et al. High-Intensity Interval Training After Stroke: An Opportunity to Promote Functional Recovery, 
Cardiovascular Health, and Neuroplasticity. Neurorehabil Neural Repair. 2018;32(6-7):54356. Epub 2018/04/21. doi: 10.1177/1545968318766663. PubMed PMID: 29676956.

81. King M, Kelly LP, Wallack EM, Hasan SMM, Kirkland MC, Curtis ME, et al. Serum levels of insulin-like growth factor-1 and brain-derived neurotrophic factor as potential recovery biomarkers in stroke. Neurol Res. 2019;41(4):354-63. Epub 2019/01/09. doi: 10.1080/01616412.2018.1564451. PubMed PMID: 30620251.

82. Lipsky RH, Marini AM. Brain-derived neurotrophic factor in neuronal survival and behavior-related plasticity. Ann N Y Acad Sci. 2007;1122:130-43. Epub 2007/12/14. doi: 10.1196/annals.1403.009. PubMed PMID: 18077569.

83. Mattson MP. Glutamate and neurotrophic factors in neuronal plasticity and disease. Ann N Y Acad Sci. 2008;1144:97-112. Epub 2008/12/17. doi: 10.1196/annals.1418.005. PubMed PMID: 19076369; PubMed Central PMCID: PMCPMC2614307.

84. Ploughman M, Windle V, MacLellan CL, White N, Dore JJ, Corbett D. Brain-derived neurotrophic factor contributes to recovery of skilled reaching after focal ischemia in rats. Stroke. 2009;40(4):1490-5. Epub 2009/01/24. doi: 10.1161/STROKEAHA.108.531806. PubMed PMID: 19164786.

85. Cowansage KK, LeDoux JE, Monfils MH. Brain-derived neurotrophic factor: a dynamic gatekeeper of neural plasticity. Curr Mol Pharmacol. 2010;3(1):12-29. Epub 2009/12/25. doi: 10.2174/1874467211003010012. PubMed PMID: 20030625.

86. Chen J, Zhang C, Jiang H, Li Y, Zhang L, Robin A, et al. Atorvastatin induction of VEGF and BDNF promotes brain plasticity after stroke in mice. J Cereb Blood Flow Metab. 2005;25(2):281-90. Epub 2005/01/29. doi: 10.1038/sj.jcbfm.9600034. PubMed PMID: 15678129; PubMed Central PMCID: PMCPMC2804085. 
87. Kim MW, Bang MS, Han TR, Ko YJ, Yoon BW, Kim JH, et al. Exercise increased BDNF and trkB in the contralateral hemisphere of the ischemic rat brain. Brain Res. 2005;1052(1):16-21. Epub 2005/08/02. doi: 10.1016/j.brainres.2005.05.070. PubMed PMID: 16054599.

88. Vaynman S, Ying Z, Gomez-Pinilla F. Hippocampal BDNF mediates the efficacy of exercise on synaptic plasticity and cognition. Eur J Neurosci. 2004;20(10):2580-90. Epub 2004/11/19. doi: 10.1111/j.1460-9568.2004.03720.x. PubMed PMID: 15548201.

89. Chen J, Zacharek A, Zhang C, Jiang H, Li Y, Roberts C, et al. Endothelial nitric oxide synthase regulates brain-derived neurotrophic factor expression and neurogenesis after stroke in mice. J Neurosci. 2005;25(9):2366-75. Epub 2005/03/05. doi: 10.1523/JNEUROSCI.507104.2005. PubMed PMID: 15745963; PubMed Central PMCID: PMCPMC2791344.

90. Madinier A, Bertrand N, Mossiat C, Prigent-Tessier A, Beley A, Marie C, et al. Microglial involvement in neuroplastic changes following focal brain ischemia in rats. PLoS One. 2009;4(12):e8101. Epub 2009/12/04. doi: 10.1371/journal.pone.0008101. PubMed PMID: 19956568; PubMed Central PMCID: PMCPMC2779656.

91. Schabitz WR, Steigleder T, Cooper-Kuhn CM, Schwab S, Sommer C, Schneider A, et al. Intravenous brain-derived neurotrophic factor enhances poststroke sensorimotor recovery and stimulates neurogenesis. Stroke. 2007;38(7):2165-72. Epub 2007/05/19. doi:

10.1161/STROKEAHA.106.477331. PubMed PMID: 17510456.

92. Fritsch B, Reis J, Martinowich K, Schambra HM, Ji Y, Cohen LG, et al. Direct current stimulation promotes BDNF-dependent synaptic plasticity: potential implications for motor learning. Neuron. 2010;66(2):198-204. Epub 2010/05/04. doi: 10.1016/j.neuron.2010.03.035. PubMed PMID: 20434997; PubMed Central PMCID: PMCPMC2864780. 
PAGE 52

93. Clarkson AN, Overman JJ, Zhong S, Mueller R, Lynch G, Carmichael ST. AMPA receptorinduced local brain-derived neurotrophic factor signaling mediates motor recovery after stroke. J Neurosci. 2011;31(10):3766-75. Epub 2011/03/11. doi: 10.1523/JNEUROSCI.578010.2011. PubMed PMID: 21389231; PubMed Central PMCID: PMCPMC4698878.

94. Cook DJ, Nguyen C, Chun HN, I LL, Chiu AS, Machnicki M, et al. Hydrogel-delivered brain-derived neurotrophic factor promotes tissue repair and recovery after stroke. J Cereb Blood Flow Metab. 2017;37(3):1030-45. Epub 2016/05/14. doi:

10.1177/0271678X16649964. PubMed PMID: 27174996; PubMed Central PMCID: PMCPMC5363479.

95. Prieto GA, Snigdha S, Baglietto-Vargas D, Smith ED, Berchtold NC, Tong L, et al. Synapsespecific IL-1 receptor subunit reconfiguration augments vulnerability to IL-1beta in the aged hippocampus. Proc Natl Acad Sci U S A. 2015;112(36):E5078-87. Epub 2015/08/26. doi: 10.1073/pnas.1514486112. PubMed PMID: 26305968; PubMed Central PMCID:

\section{PMCPMC4568670.}

96. Colie S, Sarroca S, Palenzuela R, Garcia I, Matheu A, Corpas R, et al. Neuronal p38alpha mediates synaptic and cognitive dysfunction in an Alzheimer's mouse model by controlling beta-amyloid production. Sci Rep. 2017;7:45306. Epub 2017/04/01. doi: 10.1038/srep45306. PubMed PMID: 28361984; PubMed Central PMCID: PMCPMC5374488.

97. Chollet F. Pharmacologic approaches to cerebral aging and neuroplasticity: insights from the stroke model. Dialogues Clin Neurosci. 2013;15(1):67-76. Epub 2013/04/12. PubMed PMID: 23576890; PubMed Central PMCID: PMCPMC3622470.

98. George PM, Steinberg GK. Novel Stroke Therapeutics: Unraveling Stroke Pathophysiology and Its Impact on Clinical Treatments. Neuron. 2015;87(2):297-309. Epub 2015/07/17. doi: 
PAGE 53

10.1016/j.neuron.2015.05.041. PubMed PMID: 26182415; PubMed Central PMCID:

PMCPMC4911814.

99. Small SL, Buccino G, Solodkin A. Brain repair after stroke--a novel neurological model. Nat

Rev Neurol. 2013;9(12):698-707. Epub 2013/11/13. doi: 10.1038/nrneurol.2013.222.

PubMed PMID: 24217509; PubMed Central PMCID: PMCPMC5549938.

100. Balkaya M, Krober JM, Rex A, Endres M. Assessing post-stroke behavior in mouse models of focal ischemia. J Cereb Blood Flow Metab. 2013;33(3):330-8. Epub 2012/12/13. doi:

10.1038/jcbfm.2012.185. PubMed PMID: 23232947; PubMed Central PMCID:

PMCPMC3587814. 


\section{$A$ Vehicle $\boxminus$ NFMD $1.5 \mathrm{mg} / \mathrm{kg}$ 四 NFMD $4.5 \mathrm{mg} / \mathrm{kg}$}

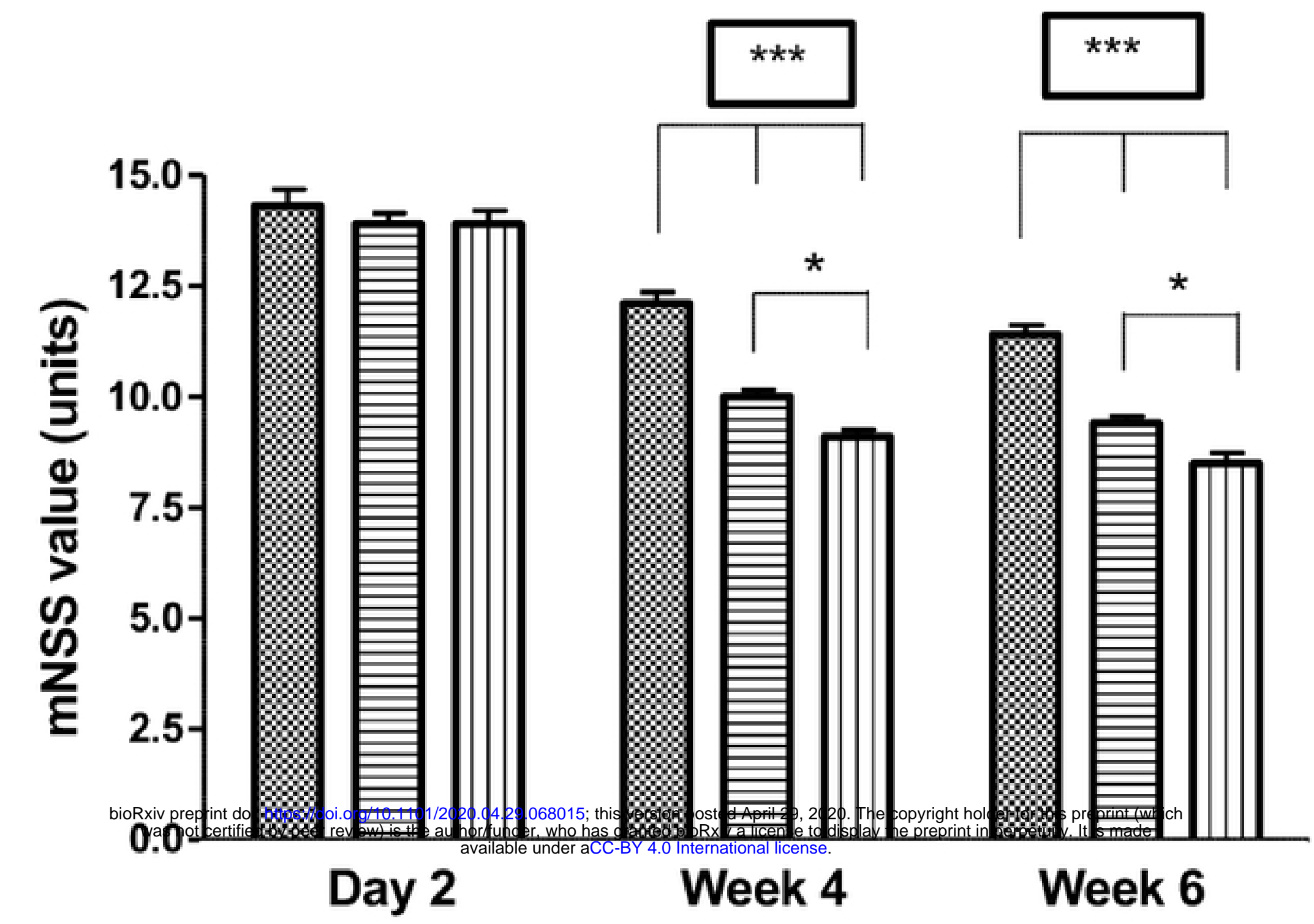

B Vehicle $巴$ NFMD $1.5 \mathrm{mg} / \mathrm{kg}$ 血 NFMD $4.5 \mathrm{mg} / \mathrm{kg}$

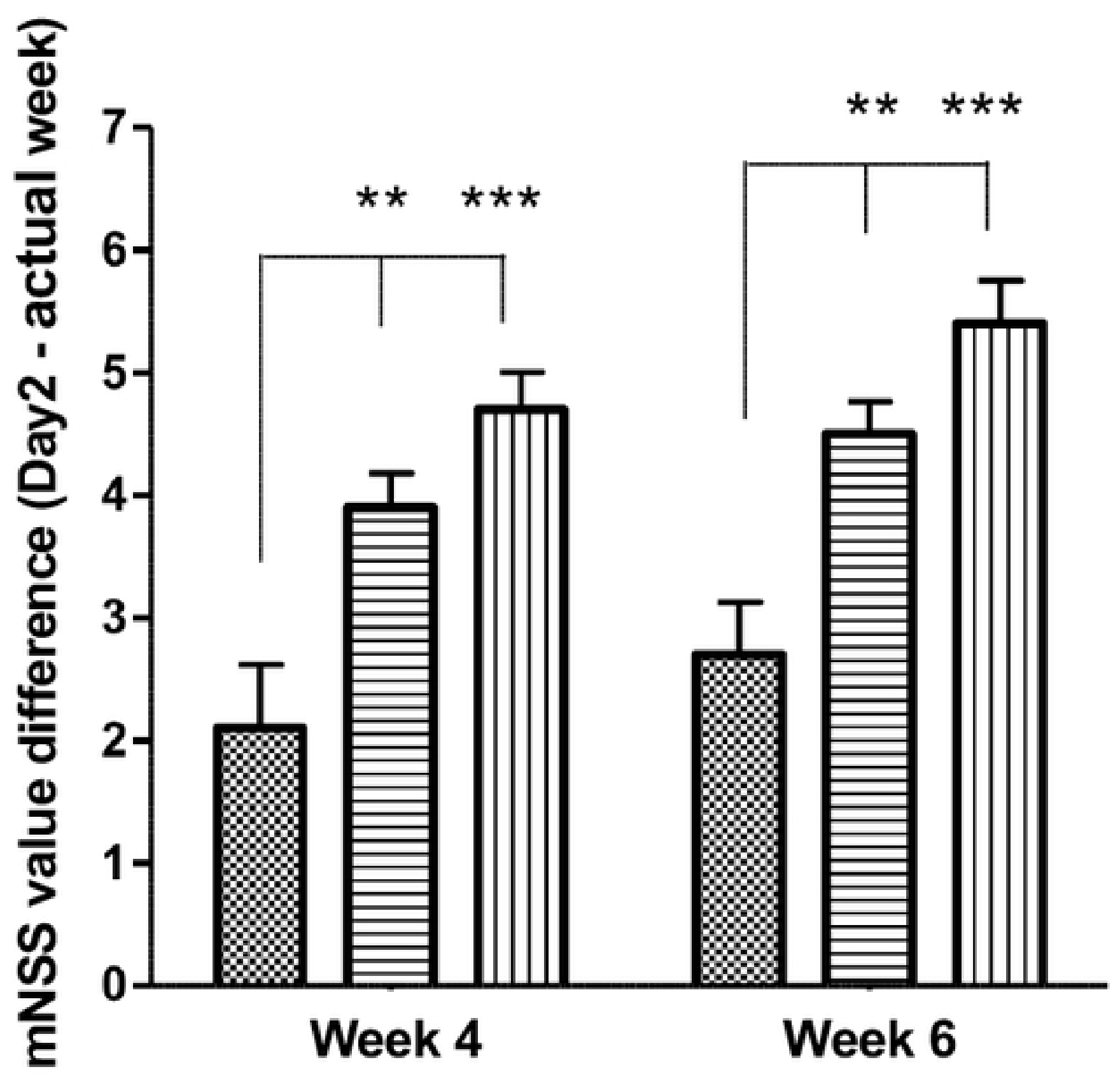

Figure 
$\Delta \quad$ Vehicle $\boxminus N F M D 1.5 \mathrm{mg} / \mathrm{kg}$ 四NFD $4.5 \mathrm{mg} / \mathrm{kg}$

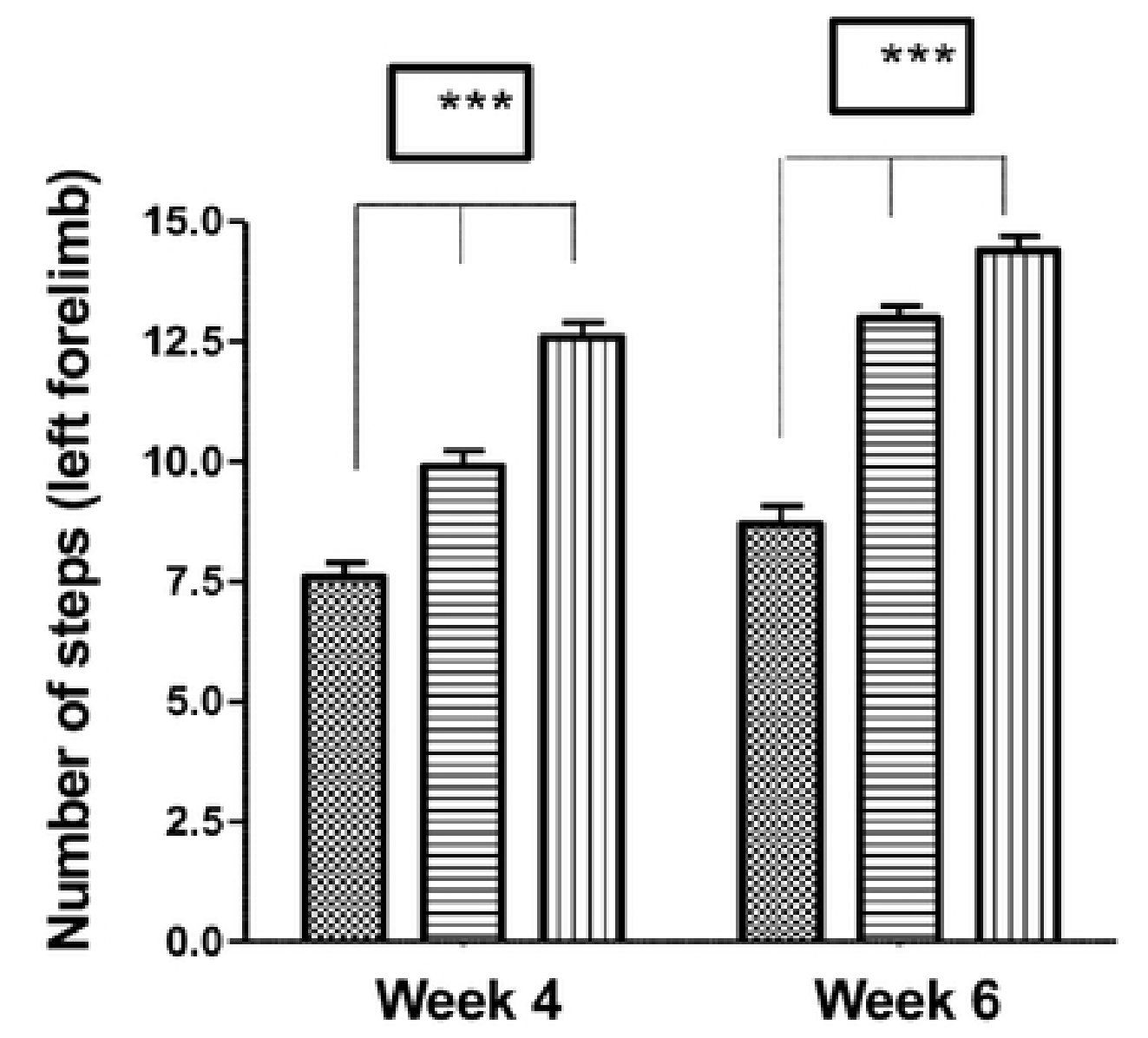

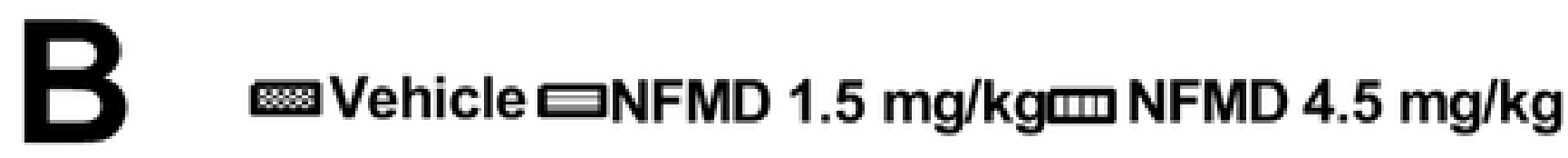

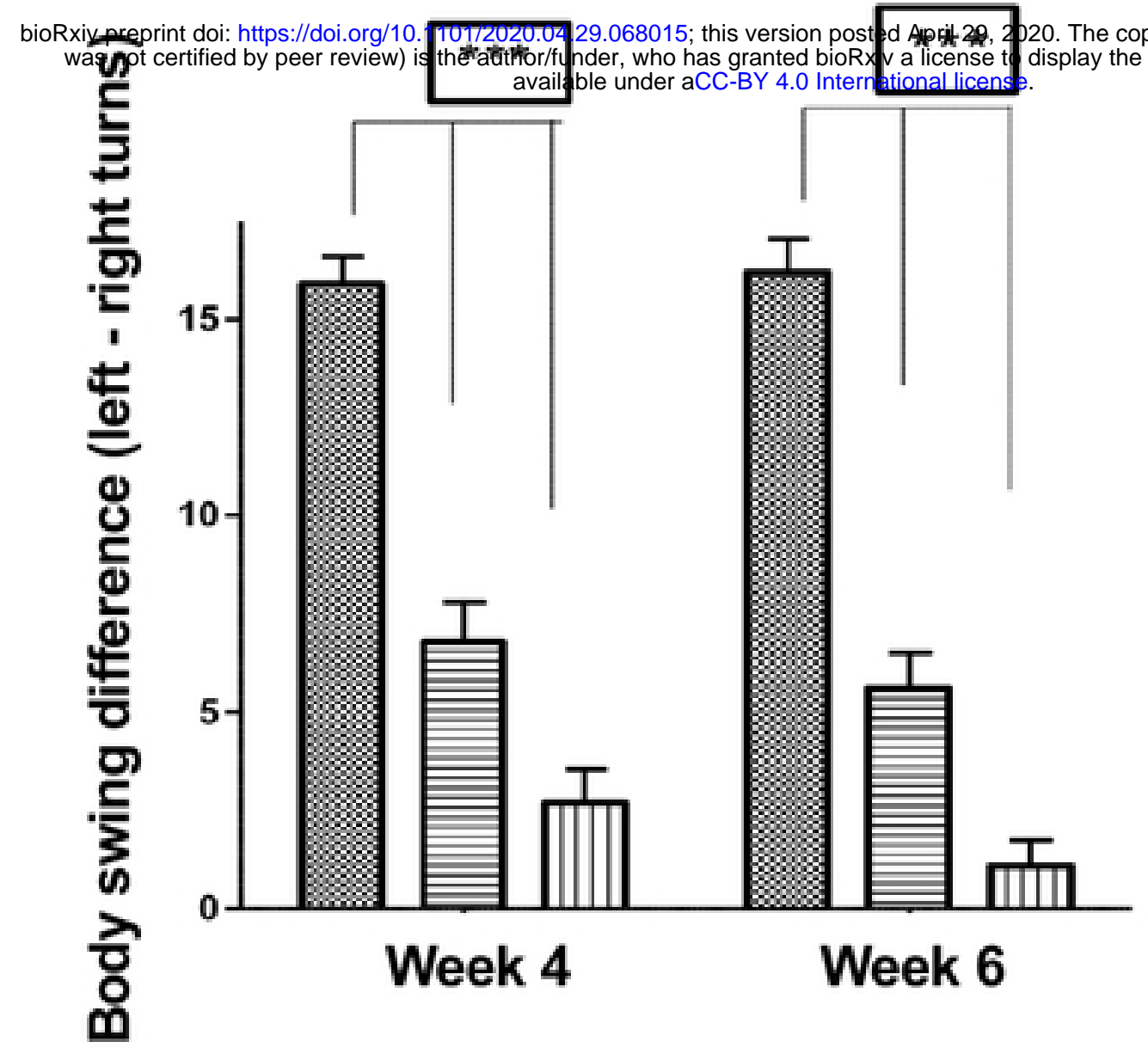

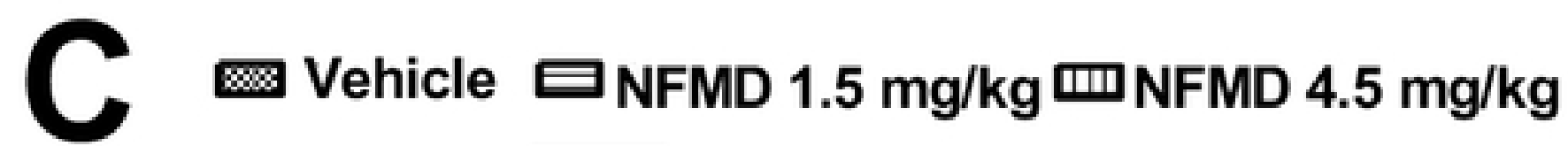

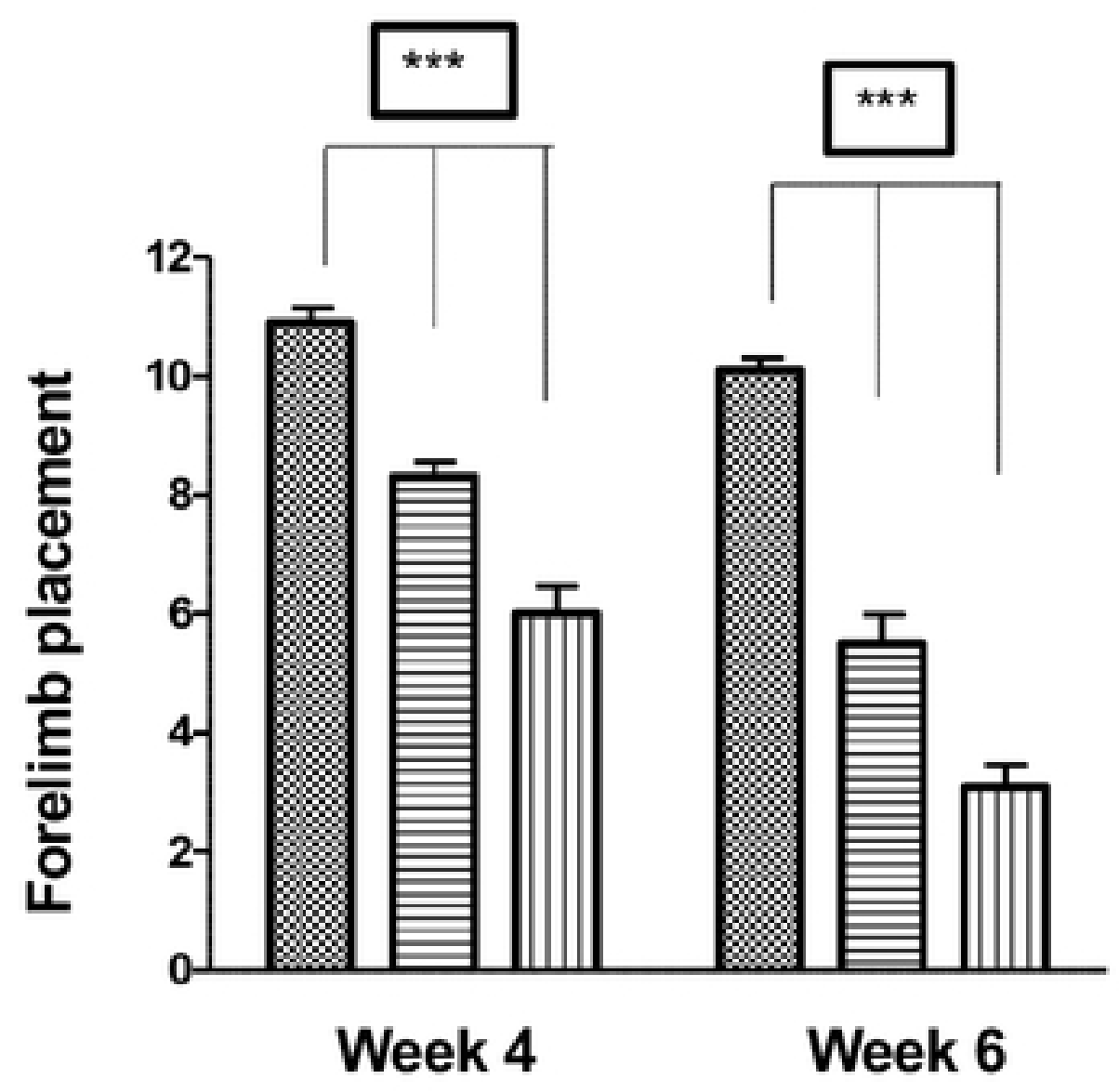

Figure 

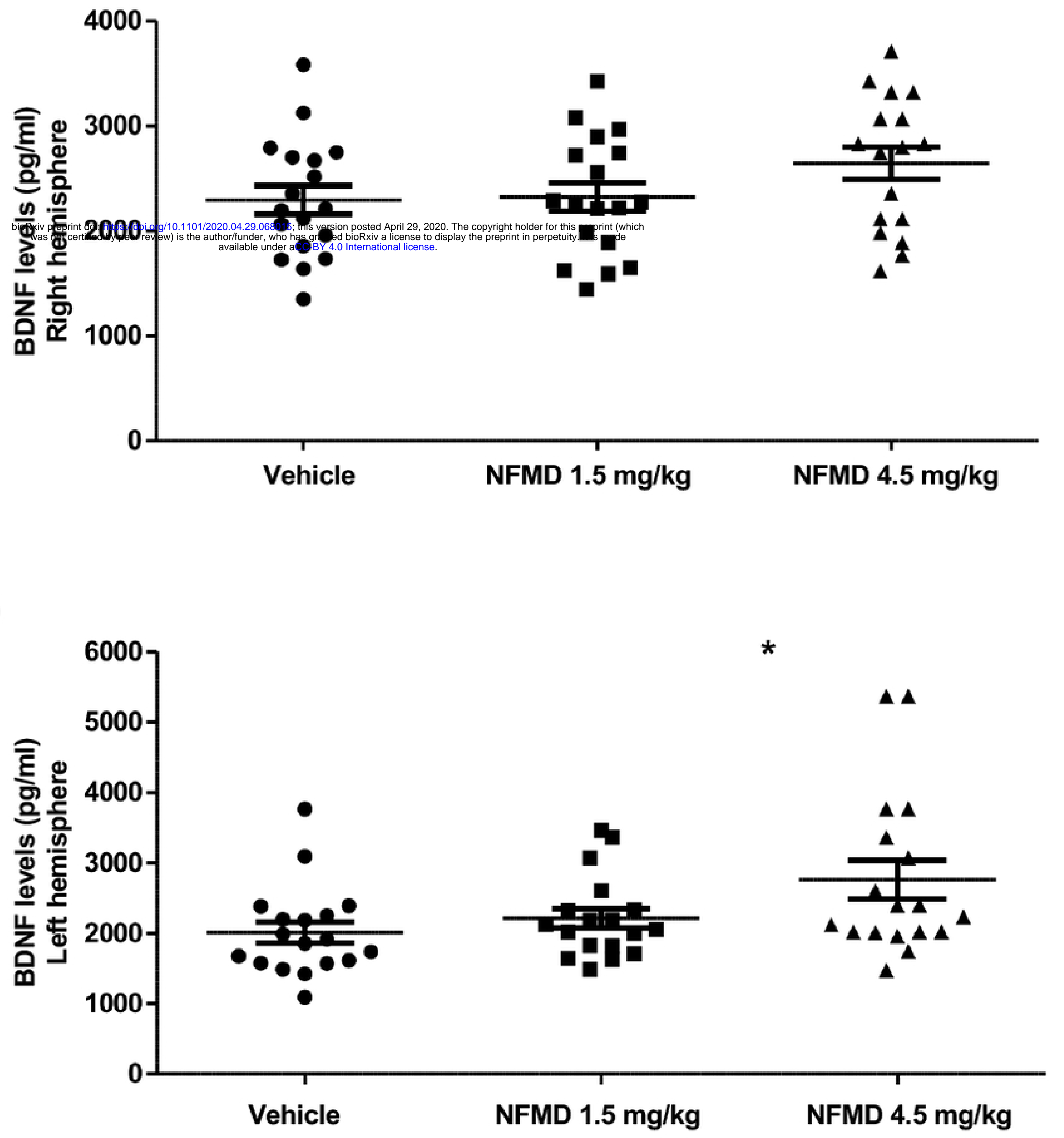

Figure 